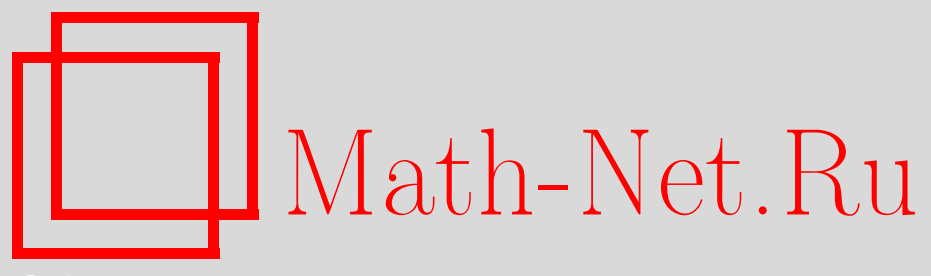

А. В. Дымов, Диссипативные эффекты в одной линейной лагранжевой системе с бесконечным числом степеней свободы, Изв. РАН. Сер. матем., 2012, том 76, выпуск $6,45-80$

DOI: https://doi.org/10.4213/im7796

Использование Общероссийского математического портала Math-Net.Ru подразумевает, что вы прочитали и согласны с пользовательским соглашением http: //www . mathnet.ru/rus/agreement

Параметры загрузки:

IP : 52.87 .193 .239

26 апреля 2023 г., 09:16:23

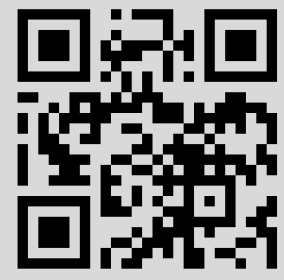




\title{
А. В. Дымов \\ Диссипативные эффекты \\ в одной линейной лагранжевой системе с бесконечным числом степеней свободы
}

\begin{abstract}
Рассматривается задача потенциального взаимодействия конечномерной и бесконечномерной линейных лагранжевых систем (системы линейных осцилляторов и термостата). Изучается финальная динамика системы. При выполнении естественных предположений она оказывается очень простой и описывается в явном виде, так как несмотря на сохранение энергии и лагранжеву природу системы термостат обеспечивает некоторое эффективное трение. Применяются методы статьи [1], в которой исследована финальная динамика конечномерной подсистемы в случае, когда она имеет одну степень свободы и обладает линейным или, при некоторых дополнительных предположениях, полиномиальным потенциалом. Рассмотрен случай, когда конечномерная подсистема обладает произвольным числом степеней свободы и линейным потенциалом. Исследуется финальная динамика как системы осцилляторов, так и термостата. Все необходимые утверждения из [1] приведены с доказательствами, адаптированными для рассматриваемого случая.

Библиография: 12 наименований.
\end{abstract}

Ключевые слова: лагранжевы системы, гамильтоновы системы, системы с бесконечным числом степеней свободы, финальная динамика.

\section{§ 1. Введение}

Задача взаимодействия конечномерной и бесконечномерной гамильтоновых систем всегда привлекала внимание физиков и специалистов по динамике. В отличие от конечномерной динамики, несмотря на сохранение энергии в таких системах обычно появляется некоторое эффективное трение. А именно, часть энергии становится "ненаблюдаемой" за счет того, что ее абсорбирует бесконечномерная система.

Задачи такого типа сложны, поэтому обычно приходится считать, что бесконечномерная система линейна. Она может моделироваться как термостат [2], как струна (или, в более общем случае, как система Клейна-Гордона) [3]-[6] либо как электромагнитное поле (уравнения Максвелла) [7]. Мы, как и в [1], [2], представляем ее как континуум независимых осцилляторов и называем термостатом ${ }^{1}$. Конечномерную систему мы также предполагаем линейной, но имеющей произвольную размерность.

\footnotetext{
${ }^{1}$ Такая терминология может показаться физически не совсем оправданной, но нам хотелось бы ее использовать, так как она порождает интуицию.
} 
В данном тексте предполагается, что полная энергия системы конечна. Метод, разработанный в [1] и применяемый нами, основан на том наблюдении, что конечномерная система должна осциллировать так, чтобы в термостате не возникало резонансов (их возникновение противоречит конечности энергии). Это условие влечет некоторое ограничение, налагаемое на спектр Фурье конечномерной компоненты решения. Согласно этому удается установить, что при времени, стремящемся как к $+\infty$, так и к $-\infty$, движение конечномерной подсистемы становится квазипериодическим, точнее, стремится к конечной линейной комбинации гармонических колебаний. При этом количество частот в данном квазипериодическом движении не превышает числа степеней свободы конечномерной подсистемы. Часть энергии "растворяется" в термостате, т. е. концентрируется в компоненте движения бесконечномерной подсистемы, слабо стремящейся к нулю. Финальная динамика термостата весьма схожа: его движение слабо стремится к квазипериодическому режиму с теми же собственными частотами.

Теперь перейдем к технической части изложения. Рассмотрим лагранжеву систему

$$
\frac{d}{d t} \frac{\partial \mathcal{L}_{0}}{\partial \dot{x}}-\frac{\partial \mathcal{L}_{0}}{\partial x}=0, \quad \mathcal{L}_{0}=\mathcal{L}_{0}(x, \dot{x}), \quad x \in \mathbb{R}^{n}
$$

здесь $x=\left(x_{1}, \ldots, x_{n}\right)^{T}$, где через $T$ обозначено транспонирование, и лагранжиан $\mathcal{L}_{0}$ равен разности между кинетической и потенциальной энергиями:

$$
\mathcal{L}_{0}(x, \dot{x})=\frac{1}{2}\langle\dot{x}, \dot{x}\rangle-\frac{1}{2}\langle B x, x\rangle .
$$

Матрица $B$ размера $n \times n$ предполагается симметричной и положительно определенной.

Под скалярным произведением $\langle$,$\rangle подразумевается стандартное евклидо-$ во скалярное произведение. В частности, $\langle B x, x\rangle=\sum_{i, j=1}^{n} b_{i j} x_{i} x_{j}$, где $b_{i j}-$ элементы матрицы $B$.

ЗАмечАниЕ 1.1. Задача с лагранжианом более общего вида

$$
\mathcal{L}_{0}(x, \dot{x})=\frac{1}{2}\langle A \dot{x}, \dot{x}\rangle-\frac{1}{2}\langle B x, x\rangle,
$$

где $A$ - положительно определенная симметричная матрица, очевидным образом сводится к рассматриваемой задаче с помощью замены переменных.

Рассмотрим другую систему, которую мы будем называть термостатом. Это бесконечномерная линейная лагранжева система с лагранжианом

$$
\mathcal{L}_{T}(\xi, \dot{\xi})=\int \frac{\rho(\nu)}{2}\left(\dot{\xi}^{2}(\nu, t)-\nu^{2} \xi^{2}(\nu, t)\right) d \nu
$$

(для краткости вместо $\int_{-\infty}^{+\infty}$ будем записывать $\int$ ). Здесь $\xi(\nu, t)$ и $\rho(\nu)-$ действительные функции. Такую систему можно трактовать как бесконечный набор независимых гармонических осцилляторов, параметризованных собственной частотой $\nu$. Физический смысл $\rho(\nu)-$ плотность массы ${ }^{2}$ осцилляторов

\footnotetext{
2 Заметим, что масса термостата может быть как конечна, так и бесконечна.
} 
с частотой $\nu$. Уравнение движения изолированного термостата имеет следующий вид:

$$
\rho(\nu)\left(\ddot{\xi}(\nu, t)+\nu^{2} \xi(\nu, t)\right)=0 .
$$

Теперь рассмотрим взаимодействие двух описанных выше систем со следующим потенциалом:

$$
V_{\mathrm{int}}=-\sum_{l=1}^{n} x_{l} \int \kappa_{l}(\nu) \xi d \nu=-\left\langle x, \int \kappa(\nu) \xi d \nu\right\rangle
$$

$x=\left(x_{1}, \ldots, x_{n}\right)^{T}, \kappa(\nu)=\left(\kappa_{1}(\nu), \ldots, \kappa_{n}(\nu)\right)^{T}, \int \kappa \xi d \nu=\left(\int \kappa_{1} \xi d \nu, \ldots, \int \kappa_{n} \xi d \nu\right)^{T}$. Такая система имеет лагранжиан $\mathcal{L}_{0}+\mathcal{L}_{T}-V_{\text {int }}$, и ее уравнения движения имеют вид

$$
\begin{gathered}
\ddot{x}+B x=\int \kappa \xi d \nu, \\
\rho\left(\ddot{\xi}+\nu^{2} \xi\right)=\langle x, \kappa\rangle .
\end{gathered}
$$

Заданы следующие начальные условия:

$$
\left.x\right|_{t=0}=x_{0},\left.\quad \xi\right|_{t=0}=\xi_{0}(\nu),\left.\quad \dot{x}\right|_{t=0}=\dot{x}_{0},\left.\quad \dot{\xi}\right|_{t=0}=\dot{\xi}_{0}(\nu) .
$$

Полная энергия системы имеет вид

$$
E(x, \xi, \dot{x}, \dot{\xi})=\frac{1}{2}\langle\dot{x}, \dot{x}\rangle+\frac{1}{2} \int \rho \dot{\xi}^{2} d \nu+\frac{1}{2}\langle B x, x\rangle+\frac{1}{2} \int \rho \nu^{2} \xi^{2} d \nu+V_{\text {int }} .
$$

Колебания системы стремятся к квазипериодическому режиму при времени, стремящемся к $\pm \infty$, за счет того, что термостат создает эффективное трение. Функция $\xi(\nu, t)$ осциллирует по переменной $\nu$ все быстрее и быстрее (см. (7.3)), вследствие чего некоторая ее компонента слабо стремится к нулю, забирая заметную часть энергии, которая "растворяется" в термостате. Данное явление напоминает превращение энергии в тепло. При этом в преобразовании Фурье функции $\xi$ по переменной $\nu$ эти осцилляции порождают волны, уходящие на бесконечность. Такой механизм поглощения энергии термостатом, фурье-дуальный к первому, можно интерпретировать как излучение.

Данный текст организован следующим образом. В $\S 2$ формулируются предположения Н1-H6, налагаемые на функции $\xi_{0}, \dot{\xi}_{0}, \rho, \kappa_{l}$. Одна часть предположений отвечает за регулярность этих функций, другая - за положительную определенность и конечность энергии $E$, которая необходима нам для доказательства теоремы 2.1 существования решения системы (1.4)-(1.6) при всех $t \in \mathbb{R}$.

Предположение Н6 несколько отличается от предположений Н1-H5. В нем налагается ограничение на вид взаимодействия между термостатом и конечномерной системой. А именно, $\kappa_{i} /\left.\sqrt{\rho}\right|_{\left(-\nu_{0}, \nu_{0}\right)}=0$ при каждом $1 \leqslant i \leqslant n$ для некоторого $\nu_{0} \geqslant 0$. При $\nu_{0}>0$ это значит, что осцилляторы термостата, колеблющиеся на частотах $\nu \in\left(-\nu_{0}, \nu_{0}\right)$, никак не влияют на динамику конечномерной системы (так как правая часть уравнения (1.5), деленного на $\sqrt{\rho}$, в точках из этого интервала обращается в нуль). На промежутке $\sigma=\mathbb{R} \backslash\left(-\nu_{0}, \nu_{0}\right)$ 
предполагается выполнение некоторых условий невырожденности: ситуация, при которой все функции $\kappa_{i} / \sqrt{\rho}$ одновременно обращаются в нуль на некотором промежутке, кроме зафиксированного нами интервала $\left(-\nu_{0}, \nu_{0}\right)$, является нетипичной. При $\nu_{0}=0$ такой интервал отсутствует. Соответственно, условия невырожденности должны быть выполнены на промежутке $\mathbb{R} \backslash\{0\}$.

Следуя [1], мы рассматриваем пространство $\mathcal{C}_{b}$ ограниченных равномерно непрерывных комплексных функций на прямой и его преобразование Фурье $\widehat{\mathcal{C}_{b}}=\mathcal{F}\left(\mathcal{C}_{b}\right)$, состоящее из обобщенных функций. Подпространство $\mathcal{C}_{0} \subset \mathcal{C}_{b}$ состоит из функций, стремящихся к нулю при аргументе, в свою очередь, стремящемся к $\pm \infty$. Для каждой функции $\widehat{\varphi} \in \widehat{\mathcal{C}}_{b}$ мы определяем сингулярный носитель $\operatorname{sing} \operatorname{supp}(\widehat{\varphi})$ следующим образом: мы говорим, что $\lambda \in \operatorname{sing} \operatorname{supp}(\widehat{\varphi})$, если для любой достаточно малой окрестности точки $\lambda$ существует регулярная (например, гладкая) функция $\hat{h}$ с носителем в этой окрестности такая, что обратное преобразование Фурье $\mathcal{F}^{-1}$ функции $\hat{h} \widehat{\varphi}$ не лежит в $\mathcal{C}_{0}$.

Также в $\S 2$ формулируется теорема 2.2. Она носит технический характер, но очень важна для исследования финальной динамики системы. Ее первая часть говорит о регулярности функций $\widehat{x}_{i}(\nu)=\mathcal{F}\left(x_{i}\right)(\nu), x_{i}=x_{i}(t)$, на промежутке $\nu \in \sigma=\mathbb{R} \backslash\left(-\nu_{0}, \nu_{0}\right)$. Это означает, что $\operatorname{sing} \operatorname{supp}\left(\widehat{x}_{i}\right) \subset\left(-\nu_{0}, \nu_{0}\right)$. Таким образом, функция $\widehat{x}_{i}$ может иметь сингулярность только на тех частотах, где термостат не влияет на конечномерную подсистему. Вторая часть теоремы 2.2 предоставляет связь между сингулярной частью $\widehat{x}_{i}$ и $\int \kappa_{i} \xi d \nu$, где последний интеграл взят из (1.4).

В $\S 3$ дано начало доказательства теоремы 2.1 (п. 3.1), но, главным образом, проведено исследование финальной динамики конечномерной системы (п. 3.2). Доказывается теорема 3.1 , утверждающая, что в случае $\nu_{0}=0$ при $t \rightarrow \pm \infty$ колебания конечномерной системы затухают, а в случае $\nu_{0}>0$ - стремятся к линейной комбинации из гармонических колебаний с частотами $\lambda_{1}, \ldots, \lambda_{k}$, лежащими на интервале $\left(-\nu_{0}, \nu_{0}\right), k \leqslant n$, или затухают, если $k=0$.

$\mathrm{B} \S 4$ для удобства изложения вынесены доказательства некоторых промежуточных утверждений, используемых в 33 .

В п. 5.1 показано, что хорошо известное уравнение Клейна-Гордона порождает термостат, т.е. оно эквивалентно системе с лагранжианом (1.3). С помощью этого наблюдения строится естественный пример системы (1.4),(1.5). В п. 5.2 разбирается пример линейной системы более общего вида, чем рассмотренная выше. Показывается, что при помощи замены переменных она сводится к рассмотренной системе.

В $\S 6$ заканчивается доказательство теоремы 2.1. В $\S 7$ дается доказательство теоремы 2.2. В начале п. 7.2 доказывается, что $L_{2}$-норма функции $\left\langle\widehat{a}, \widehat{x}^{t}\right\rangle$ равномерно ограничена (следствие 7.2), где $\widehat{a}_{i}=\frac{\kappa_{i}}{\sqrt{\rho}}, \widehat{x}_{i}^{t}-$ преобразование Фурье функции $x_{i}(\tau) \chi_{(0, t)}, \tau \in \mathbb{R}$, а $\chi_{(0, t)}$ - индикатор интервала $(0, t)$. Это является ключевым моментом в доказательстве утверждения 1) теоремы 2.2 (п. 7.2 ). Утверждение 2) теоремы 2.2 доказывается в п. 7.1.

В $\S 8$ исследуется финальная динамика термостата. Доказывается теорема 8.1, утверждающая, что движение термостата слабо стремится к такому же предельному режиму, как и конечномерная подсистема. А именно, показывается, что для любой наблюдаемой $\widehat{\varphi}(\nu)$ из некоторого класса интеграл $\int \widehat{\varphi} \xi d \nu$ 
при $t \rightarrow \pm \infty$ стремится к линейной комбинации из гармонических колебаний с теми же частотами, что и конечномерная подсистема.

В $\S 9$ дается самодостаточная теория используемых нами пространств $\mathcal{C}_{b}, \mathcal{C}_{0}$ и других, а также их преобразований Фурье. С несколько другой точки зрения она изложена в статье [8].

Выражаю огромную благодарность моему научному руководителю Д. В. Трещеву за постановку данной задачи, а также всестороннюю помощь в ее решении. Кроме того, благодарю А. А. Шкаликова за большое внимание к работе, конструктивную критику и ценные советы.

\section{§2. Технические теоремы}

2.1. Предположения $\mathrm{H1-H4} \mathrm{и} \mathrm{теорема} \mathrm{существования.} \mathrm{Сначала}$ сформулируем следующие предположения.

Н1. Функиии $\rho, \kappa_{l}$ измеримь, функиия $\rho$ неотрицателъна и $\operatorname{supp} \kappa_{l} \subset$ $\operatorname{supp} \rho$ nри всеx $l=1, \ldots, n$.

Далее мы доопределим функции $\kappa_{l} / \sqrt{\rho}$ на $\mathbb{R} \backslash \operatorname{supp} \rho$ нулем.

Н2. Интеграль $\int \frac{\kappa_{l}^{2}}{\rho \nu^{2}} d \nu$ конечны при всех $l=1, \ldots, n$.

Пусть $K=\left(k_{l m}\right)=\left(\int \frac{\kappa_{l} \kappa_{m}}{\rho \nu^{2}} d \nu\right)-$ матрица размера $n \times n$.

Н3. Матрица $B-K$ положительно определена.

В частности, отсюда следует неотрицательность энергии (1.7):

$$
E=\frac{1}{2}\langle\dot{x}, \dot{x}\rangle+\frac{1}{2} \int \rho \dot{\xi}^{2} d \nu+\frac{1}{2}\langle(B-K) x, x\rangle+\frac{1}{2} \int \rho \nu^{2}\left(\xi-\left\langle\frac{\kappa}{\rho \nu^{2}}, x\right\rangle\right)^{2} d \nu \geqslant 0 .
$$

Н4. Интеграл $\int \rho\left(\dot{\xi}_{0}^{2}+\nu^{2} \xi_{0}^{2}\right) d \nu$ конечен.

ЗАмечАниЕ 2.1. Из равенства (1.7) и предположений Н2, Н4 следует оценка $E\left(x_{0}, \xi_{0}, \dot{x}_{0}, \dot{\xi}_{0}\right)<\infty$. Действительно, с помощью (1.7) несложно убедиться в том, что $\int \kappa_{l} \xi_{0} d \nu<\infty$ при всех $l=1, \ldots, n$. Это следует из неравенства Коши-Буняковского $\left(\int \kappa_{l} \xi_{0} d \nu\right)^{2} \leqslant k_{l l} \int \rho \nu^{2} \xi_{0}^{2} d \nu$.

Теорема 2.1. Пусть предположения Н1-Н4 выполнены. Тогда существует единственное решение $x(t), \xi(\nu, t)$ уравнений (1.4)-(1.6). Оно определено nри всех $t \in \mathbb{R} u$ имеет конечную и постоянную энергию $E=$ const $<\infty$. Кроме того, решение обладает следующими свойствами:

1) функиии $x(t)=\left(x_{1}(t), \ldots, x_{n}(t)\right)$ ограничены и равномерно непрерывны;

2) бункиия $\mathcal{E}(t)=\int \rho(\nu)\left(\nu^{2} \xi^{2}(\nu, t)+\dot{\xi}^{2}(\nu, t)\right) d \nu$ ограничена.

Начало доказательства теоремы 2.1 приведено в п. 3.1, а свойства 1) и 2) доказаны в $\S 6$. 
2.2. Пространства и обозначения. Пусть $\mathcal{C}_{b}$ - пространство равномерно непрерывных ограниченных функций $\mathbb{R} \rightarrow \mathbb{C}$. Аргументом функций из $\mathcal{C}_{b}$ обычно будет время $t$. Это банахово пространство с нормой $\|\varphi\|_{\infty}=\sup |\varphi|$ в $L_{\infty}$. Пусть $\mathcal{M}$ - пространство плотностей конечных комплексных счетноаддитивных мер Радона на $\mathbb{R}$. Пространство $\mathcal{M} \subset \mathcal{C}_{b}^{*}$ содержит обобщенные функции такие, что $\mu(\tau) d \tau$ - конечная комплексная счетно-аддитивная мера Радона для любой $\mu \in \mathcal{M}$. Пространство $\mathcal{M}$ является банаховым с нормой

$$
\|\mu\|_{\mathcal{M}}=\sup _{\|\varphi\|_{\infty}=1} \mu(\varphi), \quad \mu(\varphi)=\int \varphi(\tau) \mu(\tau) d \tau, \quad \varphi \in \mathcal{C}_{b}, \quad \mu \in \mathcal{M},
$$

и, очевидно, содержит $L_{1}=L_{1}(\mathbb{R})$.

Обозначим через $\mathcal{F}$ преобразование Фурье

$$
\mathcal{F}(\psi)=\widehat{\psi}(\lambda)=\int e^{-i \lambda \tau} \psi(\tau) d \tau, \quad \mathcal{F}^{-1}(\widehat{\psi})=\psi(\tau)=\frac{1}{2 \pi} \int e^{i \lambda \tau} \widehat{\psi}(\lambda) d \lambda .
$$

Напомним формулу преобразования Фурье от произведения:

$$
2 \pi \mathcal{F}(\varphi \psi)=\mathcal{F} \varphi * \mathcal{F} \psi, \quad \mathcal{F}^{-1}(\widehat{\varphi} \widehat{\psi})=\mathcal{F}^{-1} \widehat{\varphi} * \mathcal{F}^{-1} \widehat{\psi}
$$

где $*$, как обычно, обозначает свертку.

Далее мы будем обозначать функции (или обобщенные функции), зависящие от времени, через $h, \varphi, \psi, \ldots$, а их преобразование Фурье - через $\hat{h}, \widehat{\varphi}, \widehat{\psi}, \ldots$. Аналогичным образом, крышка над обозначением функционального пространства означает его преобразование Фурье.

Пусть $\mathcal{C}_{0}^{+}$- подпространство в $\mathcal{C}_{b}$, состоящее из функций, стремящихся к нулю при времени, стремящемся к $+\infty$, и $\mathcal{C}_{0}^{-} \subset \mathcal{C}_{b}-$ подпространство, состоящее из функций, стремящихся к нулю при времени, стремящемся к $-\infty$. Также будем рассматривать соответствующие пространства обобщенных функций $\widehat{\mathcal{C}_{b}}=\mathcal{F}\left(\mathcal{C}_{b}\right)$ и $\widehat{\mathcal{C}}_{0}^{ \pm}=\mathcal{F}\left(\mathcal{C}_{0}^{ \pm}\right)$. Положим $\mathcal{C}_{0}=\mathcal{C}_{0}^{+} \cap \mathcal{C}_{0}^{-}$и $\widehat{\mathcal{C}_{0}}=\mathcal{F}\left(\mathcal{C}_{0}\right)$.

Определим пространство $\widehat{\mathcal{M}}=\mathcal{F}(\mathcal{M})$. Нетрудно показать, что $\widehat{\mathcal{M}} \subset \mathcal{C}_{b}$ (см. [9]). Кроме того, $L_{1} \subset \mathcal{M}$, и по теореме Римана-Лебега $\mathcal{F}\left(L_{1}\right) \subset \mathcal{C}_{0}$.

Далее для удобства записи будем использовать следующие обозначения: $\mathcal{C}_{b}^{n}-$ пространство, состоящее из элементов вида $\left(\varphi_{1}, \ldots, \varphi_{n}\right)^{T}$, где $\varphi_{l} \in \mathcal{C}_{b}$ при всех $l=1, \ldots, n$, и аналогично определяются пространства $\mathcal{C}_{0}^{n}, \mathcal{C}_{0}^{n \pm}, \mathcal{M}^{n}$. Их преобразования Фурье $\widehat{\mathcal{C}}_{0}^{n}, \widehat{\mathcal{C}}_{0}^{n \pm}, \widehat{\mathcal{M}}^{n}$ определяются покомпонентно. Будем также использовать обозначения такого типа: $\widehat{\mu} \widehat{x}=\left(\widehat{\mu} \widehat{x}_{1}, \ldots, \widehat{\mu} \widehat{x}_{n}\right)^{T}$ для $\widehat{\mu} \in \widehat{\mathcal{M}}$ и $\widehat{x} \in \widehat{\mathcal{C}}_{b}^{n}$.

Обозначим ${ }^{3}$

$$
\begin{array}{r}
\widehat{a}_{l}(\nu)=\frac{\kappa_{l}(\nu)}{\sqrt{\rho(\nu)}, \quad \widehat{w}_{l m}(\nu)=\frac{2 \pi \widehat{a}_{l}(\nu) \widehat{a}_{m}(\nu)}{i \nu},} \\
w_{\diamond l m}^{ \pm}(\tau)= \begin{cases}\frac{ \pm\left(w_{l m}(\tau)-w_{l m}(-\tau)\right)}{2}, & \text { если } \pm \tau>0, \\
0, & \text { если } \pm \tau<0,\end{cases}
\end{array}
$$

\footnotetext{
${ }^{3}$ Из условия Н5, приведенного ниже, следует, что функции $\widehat{a}_{l}, \widehat{w}_{l m}$ непрерывны.
} 
где $w_{l m}=\mathcal{F}^{-1}\left(\widehat{w}_{l m}\right)$. Стандартным вычислением можно показать, что (см. п. 7.1 далее)

$$
\widehat{w}_{\diamond l m}^{ \pm}(\nu)= \pm \frac{1}{4}\left(\widehat{w}_{l m}(\nu)-\widehat{w}_{l m}(-\nu)\right)+\frac{1}{2 \pi i} \text { v.p. } \int \frac{\lambda \widehat{w}_{l m}(\lambda) d \lambda}{\nu^{2}-\lambda^{2}} .
$$

Кроме того, будут приняты следующие обозначения: $\widehat{w}_{l}=\left(\widehat{w}_{1 l}, \ldots, \widehat{w}_{n l}\right)^{T}$, аналогично определяются $w_{l}, \widehat{w}_{\diamond l}^{ \pm}, w_{\diamond l}^{ \pm}, \widehat{a}$.

Также будут использоваться матрица $\widehat{W}=\left(\widehat{w}_{l m}\right)$ и матрица $\widehat{W}_{\diamond}^{\star}=\left(\widehat{w}_{\diamond l m}^{\star}\right)$, где $\star \in\{+,-\}$.

ЗАмечАНИЕ 2.2. Из условия Н2 следует, что $\frac{\widehat{a}_{l}}{\nu} \in L_{2}(\mathbb{R})$ для $l=1, \ldots, n$.

2.3. Предположения H5, H6. Сформулируем здесь остальные предположения.

H5. Функции $\widehat{a}_{l}$ и $\widehat{w}_{l m}$ принадлежат $\widehat{\mathcal{M}}$.

Пусть $\widehat{V}^{\star}(\nu)$ - семейство симметричных матриц размера $n \times n$,

$$
\widehat{V}^{\star}(\nu)=B-\nu^{2} I-\widehat{W}_{\diamond}^{\star}(\nu),
$$

где $I-$ единичная матрица, $\star \in\{+,-\}$.

H6. Для каждого $\star \in\{+,-\}$ выполнены следующие условия:

1) на интервале ${ }^{4}\left(-\nu_{0}, \nu_{0}\right)$ выполнено $\left.\widehat{a}_{l}\right|_{\left(-\nu_{0}, \nu_{0}\right)}=\left.\widehat{w}_{l m}\right|_{\left(-\nu_{0}, \nu_{0}\right)}=0,1 \leqslant$ $l, m \leqslant n$;

2) на множестве $\sigma=\mathbb{R} \backslash\left(-\nu_{0}, \nu_{0}\right)$ уравнение $\operatorname{det} \widehat{V}^{\star}(\nu)=0$ имеет только изолированные нули первого порядка;

3) на множестве $\sigma$ для каждого решения $\nu$ уравнения $\operatorname{det} \widehat{V}^{\star}(\nu)=0$ выполнено $\widehat{a}(\nu) \notin \operatorname{Im} \widehat{V}^{\star}(\nu)$;

4) $\operatorname{det} \widehat{V}^{\star}\left( \pm \nu_{0}\right) \neq 0$.

ЗАмЕчание 2.3. Невыполнение предположения Н6,3) означает, что одновременно $\operatorname{det} \widehat{V}^{\star}(\nu)=0$ и вектор $\widehat{a}(\nu)$ принадлежит $(n-1)$-мерному подпространству $\operatorname{Im} \widehat{V}^{\star}(\nu)$. Это легко разрушить малой деформацией системы.

Интервал $\left(-\nu_{0}, \nu_{0}\right)$ характерен тем, что на нем конечномерная подсистема не влияет на динамику термостата, так как правая часть уравнения (1.5), деленного на $\sqrt{\rho}$, обращается в нуль. И наоборот, множество $\sigma=\mathbb{R} \backslash\left(-\nu_{0}, \nu_{0}\right)$ параметризует осцилляторы, лежащие в термостате, оказывающие существенное влияние на динамику конечномерной подсистемы и системы в целом. Поэтому множество $\sigma$ естественно назвать спектром термостата.

Отметим, что при изучении финальной динамики системы окажется, что если $\nu_{0} \neq 0$, то движение конечномерной подсистемы стремится к линейной комбинации гармонических колебаний с частотами $\lambda_{l} \in\left(-\nu_{0}, \nu_{0}\right)$. Если $\nu_{0}=0$, то ее колебания затухают. Динамика термостата окажется такой же, но в слабом смысле.

\footnotetext{
${ }^{4}$ Случай пустого интервала $\nu_{0}=0$ не исключается.
} 
ПреДЛОЖеНИЕ 2.1. Пустъ выполненъ предположения Н2 и Н5. Тогда:

1) $w_{\diamond l m}^{ \pm} \in \mathcal{M}$

2) $\widehat{W}_{\diamond}^{ \pm}(0)=K$, где $K-$ матрица, определенная в Н3.

ДокаЗАтельство. Согласно Н5 имеем $w_{l m} \in \mathcal{M}$. Поэтому $w_{\diamond l m}^{ \pm}$также лежат в $\mathcal{M}$. Из замечания 2.2 следует, что $\widehat{w}_{l m}(0)=0$. Далее, утверждение 2) следует из (2.3).

2.4. Сингулярный носитель и теорема 2.2. Пусть $\widehat{\varphi} \in \widehat{\mathcal{C}}_{b}, \lambda \in \mathbb{R}$. Будем говорить, что $\lambda \notin \operatorname{sing} \operatorname{supp}^{+}(\widehat{\varphi})$, если существует интервал $I \ni \lambda$ такой, что для любой $\widehat{\mu} \in \widehat{\mathcal{M}}, \operatorname{supp} \widehat{\mu} \subset I$, имеем $\widehat{\mu} \widehat{\varphi} \in \widehat{\mathcal{C}}_{0}^{+}$. Аналогично определим $\operatorname{sing} \operatorname{supp}^{-}(\widehat{\varphi})$ и $\operatorname{sing} \operatorname{supp}(\widehat{\varphi})=\operatorname{sing} \operatorname{supp}^{+}(\widehat{\varphi}) \cup \operatorname{sing} \operatorname{supp}^{-}(\widehat{\varphi})$.

Ясно, что $\operatorname{sing} \operatorname{supp}^{ \pm}(\widehat{\varphi})$ и $\operatorname{sing} \operatorname{supp}(\widehat{\varphi})-$ замкнутые множества в $\mathbb{R}$.

Для $\widehat{\varphi} \in \widehat{\mathcal{C}}_{b}^{n}$ определим $\operatorname{sing} \operatorname{supp}(\widehat{\varphi})=\bigcup_{j=1}^{n} \operatorname{sing} \operatorname{supp}\left(\widehat{\varphi}_{j}\right)$. Аналогично определяются sing $\operatorname{supp}^{ \pm}(\widehat{\varphi})$.

ПримеР 2.1. Покажем, что $\operatorname{sing} \operatorname{supp} \delta\left(\lambda-\lambda_{0}\right)=\lambda_{0}$. Действительно, для любой функции $\widehat{\mu} \in \widehat{\mathcal{M}}$ выполнено $\widehat{\mu} \delta\left(\lambda-\lambda_{0}\right)=\widehat{\mu}\left(\lambda_{0}\right) \delta\left(\lambda-\lambda_{0}\right)$. Пусть $\widehat{\mu}\left(\lambda_{0}\right) \neq 0$. С учетом того, что $\mathcal{F}^{-1}\left(\widehat{\mu}\left(\lambda_{0}\right) \delta\left(\lambda-\lambda_{0}\right)\right)=\frac{\widehat{\mu}\left(\lambda_{0}\right)}{2 \pi} e^{-i \lambda_{0} t} \notin \mathcal{C}_{0}$, получаем $\operatorname{sing} \operatorname{supp} \delta\left(\lambda-\lambda_{0}\right)=\lambda_{0}$.

Обозначим $\varphi(t)=\int \kappa(\nu) \xi(\nu, t) d \nu$.

ТЕорема 2.2. Пустъ выполнены предположения Н1-Н6. Тогда преобразования Фуръе вектор-функиий $\widehat{x}=\mathcal{F}(x), \widehat{\varphi}=\mathcal{F}(\varphi)$ таковы, что:

1) $\operatorname{sing} \operatorname{supp}(\widehat{x}) \subset\left(-\nu_{0}, \nu_{0}\right)$;

2) $\widehat{\varphi}=\widehat{W}_{\diamond}^{+} \widehat{x}+\widehat{\vartheta}^{+}=\widehat{W}_{\diamond}^{-} \widehat{x}+\widehat{\vartheta}^{-}$для некоторьх $\widehat{\vartheta}^{ \pm} \in \widehat{\mathcal{C}}_{0}^{n \pm}$.

Мы докажем теорему 2.2 в $\S 7$.

Физический смысл утверждения 1 ) теоремы 2.2 - регулярность $\widehat{x}$ вне интервала $\left(-\nu_{0}, \nu_{0}\right)$. Как уже упоминалось, этот интервал характерен тем, что на нем $\left.\widehat{a}_{l}\right|_{\left(-\nu_{0}, \nu_{0}\right)}=0$ при всех $l=1, \ldots, n$ и правая часть уравнения $(1.5)$, деленного на $\sqrt{\rho}$ :

$$
\sqrt{\rho}\left(\ddot{\xi}+\nu^{2} \xi\right)=\langle x, \widehat{a}\rangle,
$$

равна нулю.

Если в правой части уравнения (2.5) есть сингулярность (формально говоря, существует $\lambda \in \operatorname{sing} \operatorname{supp}\langle\widehat{x}, \widehat{a}\rangle)$, то при $\nu=\lambda, \rho(\lambda) \neq 0$ возникнет резонанс, а это противоречит конечности энергии.

Условие регулярности $\langle\widehat{x}, \widehat{a}\rangle$ влечет одно из двух следствий: или $\widehat{x}$ регулярен, или некоторые компоненты $\widehat{x}$ имеют сингулярность, а в сумме $\langle\widehat{x}, \widehat{a}\rangle$ она сокращается. Такое сокращение - очень редкое событие. На множестве $\sigma$ мы его исключаем с помощью предположения Н6, 2)-4).

Интервал $\left(-\nu_{0}, \nu_{0}\right)$ носит выделенный характер, $\left.\widehat{a}_{l}\right|_{\left(-\nu_{0}, \nu_{0}\right)}=0$ при всех $l=$ $1, \ldots, n$. Поэтому $\widehat{x}$ на нем может иметь сингулярность.

Утверждение 2) теоремы 2.2 дает возможность выписать уравнение движения конечномерной подсистемы с точностью до $\mathcal{C}_{0}^{ \pm}$: уравнение $(1.4)$ принимает вид

$$
\ddot{x}+B x=W_{\diamond}^{ \pm} * x+\vartheta^{ \pm}, \quad \vartheta^{ \pm} \in \mathcal{C}_{0}^{ \pm},
$$


где $W_{\diamond}^{ \pm} * x$ - свертка матрицы с вектором, производящаяся по обычному правилу умножения матрицы на вектор. Это уравнение дает необходимое условие существования сингулярности в точке $\lambda$ (см. п. 7.2, ч. 3 доказательства утверждения 1) теоремы 2.2).

\section{§3. Финальная динамика системы}

В настоящем параграфе мы докажем утверждение 1) теоремы 2.1. Однако главной задачей данного параграфа является исследование финальной динамики $x$-компоненты системы (1.4)-(1.6).

3.1. Начало доказательства теоремы 2.1. Положим $\eta=\sqrt{\rho} \xi, \widehat{a}=$ $\kappa / \sqrt{\rho}$. Систему (1.4)-(1.6) можно записать в виде

$$
\begin{gathered}
\dot{x}=y, \quad \dot{\eta}=\zeta, \quad \dot{y}=-B x+\int \widehat{a} \eta d \nu, \quad \dot{\zeta}=-\nu^{2} \eta+\langle\widehat{a}, x\rangle, \\
\left.x\right|_{t=0}=x_{0},\left.\quad \eta\right|_{t=0}=\eta_{0}(\nu),\left.\quad y\right|_{t=0}=y_{0},\left.\quad \zeta\right|_{t=0}=\zeta_{0}(\nu) .
\end{gathered}
$$

Рассмотрим гильбертово пространство $\mathcal{H}$ с элементами $\psi=\left(\begin{array}{l}\psi_{1} \\ \psi_{2}\end{array}\right)=\left(\begin{array}{ll}x & \eta(\nu) \\ y & \zeta(\nu)\end{array}\right)$, $\eta(\nu), \zeta(\nu): \mathbb{R} \rightarrow \mathbb{C}$, и скалярным произведением, заданным формой энергии (1.7),

$$
\begin{aligned}
(\psi, \varphi)_{\mathcal{H}}=\left(\left(\begin{array}{l}
\psi_{1} \\
\psi_{2}
\end{array}\right)\right. & \left.,\left(\begin{array}{l}
\varphi_{1} \\
\varphi_{2}
\end{array}\right)\right)_{\mathcal{H}}=\left\langle y^{\psi}, \bar{y}^{\varphi}\right\rangle+\int \zeta^{\psi} \bar{\zeta}^{\varphi} d \nu+\left\langle B x^{\psi}, \bar{x}^{\varphi}\right\rangle \\
& +\int \nu^{2} \eta^{\psi} \bar{\eta}^{\varphi} d \nu-\left\langle x^{\psi}, \int \widehat{a} \bar{\eta}^{\varphi} d \nu\right\rangle-\left\langle\int \widehat{a} \eta^{\psi} d \nu, \bar{x}^{\varphi}\right\rangle .
\end{aligned}
$$

Скалярное произведение (3.3) положительно определено, так как его можно переписать в виде

$$
\begin{aligned}
(\psi, \varphi)_{\mathcal{H}}=\left\langle y^{\psi}, \bar{y}^{\varphi}\right\rangle+\int \zeta^{\psi} \bar{\zeta}^{\varphi} d \nu & +\left\langle(B-K) x^{\psi}, \bar{x}^{\varphi}\right\rangle \\
& +\int\left(\nu \eta^{\psi}-\left\langle\frac{\widehat{a}}{\nu}, x^{\psi}\right\rangle\right)\left(\nu \bar{\eta}^{\varphi}-\left\langle\frac{\widehat{a}}{\nu}, \bar{x}^{\varphi}\right\rangle\right) d \nu
\end{aligned}
$$

где матрица $K$ определена в предположении $\mathrm{H} 3$, а матрица $B-K$ положительна согласно Н3.

Энергия системы записывается в виде

$$
E(\psi)=\frac{1}{2}(\psi, \psi)_{\mathcal{H}}, \quad \psi=\left(\begin{array}{c}
\psi_{1} \\
\dot{\psi}_{1}
\end{array}\right)=\left(\begin{array}{cc}
x & \eta \\
\dot{x} & \dot{\eta}
\end{array}\right) .
$$

Таким образом, пространство $\mathcal{H}$ состоит из всех векторов $\psi$, на которых энергия $E(\psi)$ конечна.

Система (3.1),(3.2) может быть записана следующим образом:

$$
\begin{gathered}
\dot{\psi}=i \Omega \psi,\left.\quad \psi\right|_{t=0}=\psi_{0}, \quad \Omega \psi=\Omega\left(\begin{array}{l}
\psi_{1} \\
\psi_{2}
\end{array}\right)=\left(\begin{array}{c}
-i \psi_{2} \\
i \Lambda \psi_{1}
\end{array}\right), \\
\Lambda \psi_{1}=\Lambda(x, \eta)=\left(B x-\int \widehat{a} \eta d \nu, \nu^{2} \eta-\langle\widehat{a}, x\rangle\right) .
\end{gathered}
$$


ПРеДЛОЖениЕ 3.1. Оператор $\Omega$ является неограниченным самосопряженным оператором на $\mathcal{H}$.

Доказательство предложения 3.1 приведено в $\S 4$.

Решение системы (3.6) имеет вид

$$
\psi(t)=e^{i \Omega t} \psi_{0}, \quad \psi_{0} \in \mathcal{H},
$$

где оператор $e^{i \Omega t}$ ограничен, поскольку спектр $\Omega$ вещественный.

Включение $\psi_{0} \in \mathcal{H}$ обосновывается в следующем предложении.

ПРЕДЛОЖЕНИЕ 3.2. На решении (3.7) энергия конечна и сохраняется.

ДокАзАтельство. Покажем, что энергия конечна. Согласно (3.5) пространство $\mathcal{H}$ состоит из всех векторов $\psi$, на которых энергия конечна. Оператор $e^{i \Omega t}$ ограничен. Согласно замечанию 2.1 имеем $\psi_{0} \in \mathcal{H}$. Поэтому $\psi(t) \in \mathcal{H}$.

Покажем, что энергия сохраняется. Согласно (3.5) получаем

$$
2 E(\psi)=(\psi, \psi)_{\mathcal{H}}=\left(e^{i \Omega t} \psi_{0}, e^{i \Omega t} \psi_{0}\right)_{\mathcal{H}}=\left(e^{-i \Omega t} e^{i \Omega t} \psi_{0}, \psi_{0}\right)_{\mathcal{H}}=\left(\psi_{0}, \psi_{0}\right)_{\mathcal{H}},
$$

так как оператор $e^{-i \Omega t}$ сопряжен к оператору $e^{i \Omega t}$. Предложение доказано.

Таким образом, мы доказали первую часть теоремы 2.1 , доказательство второй части (свойства 1) и 2)) дано в 6 .

3.2. Исследование финальной динамики системы. При доказательстве следующей теоремы 3.1 мы будем находиться в рамках условия Н6, 1), согласно которому все функции $\left.\widehat{a}_{l}\right|_{\left(-\nu_{0}, \nu_{0}\right)}$ равны нулю. Поэтому поведение термостата на интервале $\left(-\nu_{0}, \nu_{0}\right)$ никак не влияет на динамику конечномерной подсистемы (которую мы и собираемся изучать). В связи с этим мы будем рассматривать сужение пространства $\mathcal{H}$ : по сравнению со старым определением мы полагаем, что для $\psi \in \mathcal{H}$ выполняется равенство

$$
\psi=\left(\begin{array}{cc}
x & \eta \\
y & \zeta
\end{array}\right),\left.\quad \eta\right|_{\left(-\nu_{0}, \nu_{0}\right)}=\left.\zeta\right|_{\left(-\nu_{0}, \nu_{0}\right)}=0 .
$$

Изменим соответствующим образом начальные условия в (3.2). Тогда согласно H6, 1) оператор $\Omega$ и система (3.6) корректно определены на таком пространстве. Все утверждения из п. 3.1 останутся в силе и для такого определения $\mathcal{H}$. Это изменение необходимо нам для доказательства предложения 4.5.

Теорема 3.1. Пусть условия Н1-Н6 выполнены. Тогда для некоторых $\alpha_{l}^{+}=\overline{\alpha_{l}^{-}} \in \mathbb{C}^{n}, l=1, \ldots, k, u k \leqslant n$ uмеем $^{5}$

$$
x(t)=\sum_{l=1}^{k}\left(\alpha_{l}^{+} e^{i \lambda_{l} t}+\alpha_{l}^{-} e^{-i \lambda_{l} t}\right)+\vartheta(t), \quad \vartheta \in \mathcal{C}_{0}^{n}, \quad 0<\lambda_{l}<\nu_{0} .
$$

ДокАзАТЕльство. Сначала сформулируем следующую лемму, доказательство которой содержится в $\ 4$.

\footnotetext{
${ }^{5}$ Если $k=0$, то считаем, что коэффициенты $\alpha_{l}$ и сумма в формуле (3.9) отсутствуют.
} 
Лемма 3.1. Спектр оператора $\Omega$ на интервале $\left(-\nu_{0}, \nu_{0}\right)$ состоит только из изолированных собственных значений в количестве, не большем $2 n$ :

$$
\left.\operatorname{spec}(\Omega)\right|_{\left(-\nu_{0}, \nu_{0}\right)}=\left\{ \pm \lambda_{1}, \pm \lambda_{2}, \ldots, \pm \lambda_{k}\right\}, \quad \lambda_{j}>0, \quad j=1, \ldots, k, \quad 0 \leqslant k \leqslant n .
$$

Согласно спектральной теореме [10] оператор $\Omega$ принимает вид $\Omega=\int \lambda d E_{\lambda}$, где $\left\{E_{\lambda}\right\}$ - соответствующее спектральное семейство. Так как $e^{i \Omega t}=\int e^{i \lambda t} d E_{\lambda}$, решение (3.7) представляется в виде

$$
\psi(t)=\int e^{i \lambda t} d E_{\lambda} \psi_{0}
$$

Пусть $e_{l}-l$-й базисный вектор в $\mathbb{C}^{n}$. Положим $\mathbf{1}_{l}=\left(\begin{array}{cc}B^{-1} e_{l} & 0 \\ 0 & 0\end{array}\right) \in \mathcal{H}$. Тогда $x$-компонента решения (3.11) представляется в виде

$$
x(t)=\int e^{i \lambda t} \widehat{\omega}(\lambda) d \lambda,
$$

где компоненты обобщенной вектор-функции $\widehat{\omega}$ удовлетворяют соотношению

$$
\widehat{\omega}_{l}(\lambda) d \lambda=d\left(E_{\lambda} \psi_{0}, \mathbf{1}_{l}\right)_{\mathcal{H}}
$$

Из (3.12) видно, что

$$
\widehat{\omega}=\frac{\widehat{x}}{2 \pi}, \quad \widehat{x}=\mathcal{F}(x) .
$$

Согласно утверждению 1$)$ теоремы 2.2 имеем $\operatorname{sing} \operatorname{supp}(\widehat{x}) \subset\left(-\nu_{0}, \nu_{0}\right)$. Coгласно определению сингулярного носителя $\operatorname{sing} \operatorname{supp}(\widehat{x})-$ замкнутое множество. Выберем $\widehat{\mu} \in \widehat{\mathcal{M}}$ такое, что:

1) $\operatorname{supp} \widehat{\mu} \in\left(-\nu_{0}, \nu_{0}\right)$;

2) $\widehat{\mu}$ равна 1 в некоторой окрестности точек $\left\{ \pm \lambda_{l}\right\}-$ собственных значений из леммы 3.1 - для всех $1 \leqslant l \leqslant n$;

3) $\left.\widehat{\mu}\right|_{\operatorname{sing} \operatorname{supp}(\widehat{x})}=1$.

Представим (3.12) в виде

$$
x(t)=\int e^{i \lambda t} \widehat{\mu} \widehat{\omega} d \lambda+\int e^{i \lambda t}(1-\widehat{\mu}) \widehat{\omega} d \lambda .
$$

Согласно лемме 3.1 выражение $d E_{\lambda} \psi_{0}$ при $\lambda \in\left(-\nu_{0}, \nu_{0}\right)$ отлично от нуля только в точках $\pm \lambda_{l}$. Поскольку $\pm \lambda_{l}$ - собственные значения, то согласно соотношению (3.13) и пп. 1), 2) в определении $\widehat{\mu}$ первый интеграл из (3.15) имеет вид

$$
\int e^{i \lambda t} \widehat{\mu} \widehat{\omega} d \lambda=\sum_{l=1}^{k}\left(\alpha_{l}^{+} e^{i \lambda_{l} t}+\alpha_{l}^{-} e^{-i \lambda_{l} t}\right),
$$

где $\alpha_{l}^{ \pm} \in \mathbb{C}^{n}$.

Рассмотрим второй интеграл в (3.15). Согласно п. 3) в определении $\widehat{\mu}$ имеем $\operatorname{sing} \operatorname{supp}(\widehat{x}) \cap \operatorname{supp}(1-\widehat{\mu})=\varnothing$. Получаем $\operatorname{sing} \operatorname{supp}(\widehat{\omega}) \cap \operatorname{supp}(1-\widehat{\mu})=\varnothing$ согласно (3.14). Применяя следствие 9.3 к каждой компоненте $\widehat{\omega}(1-\widehat{\mu})$, получаем, 
что $\widehat{\omega}(1-\widehat{\mu}) \in \widehat{\mathcal{C}}_{0}^{n}$. Следовательно,

$$
\int e^{i \lambda t}(1-\widehat{\mu}) \widehat{\omega} d \lambda=2 \pi \mathcal{F}^{-1}((1-\widehat{\mu}) \widehat{\omega}) \in \mathcal{C}_{0}^{n}
$$

Остается отметить, что $\alpha^{+}=\bar{\alpha}^{-}$, так как $x$ действителен.

Заметим, что, когда $\nu_{0}=0$, надо положить $\widehat{\mu}=0$. В формуле (3.15) первый интеграл исчезает, и колебания конечномерной подсистемы затухают.

\section{§ 4. Несколько доказательств}

В настоящем параграфе мы доказываем предложения и леммы, использованные в предыдущем параграфе. Для этого нам понадобятся несколько вспомогательных утверждений.

ЗАмЕчАниЕ 4.1. Энергию (1.7) можно представить в виде

$$
\begin{gathered}
E(\psi)=\frac{1}{2}\left(\dot{\psi}_{1}, \dot{\psi}_{1}\right)+\frac{1}{2}\left(\psi_{1}, \Lambda \psi_{1}\right), \quad\left(\psi_{1}, \psi_{1}^{\prime}\right)=\left\langle x, \bar{x}^{\prime}\right\rangle+\int_{\mathbb{R}} \eta \bar{\eta}^{\prime} d \nu \\
\psi_{1}=(x, \eta(\nu)), \quad \eta=\sqrt{\rho} \xi, \quad \Lambda\left(\psi_{1}\right)=\Lambda(x, \eta)=\left(B x-\int \widehat{a} \eta d \nu, \nu^{2} \eta-\langle\widehat{a}, x\rangle\right) .
\end{gathered}
$$

Скалярное произведение (3.3) задано формой энергии, поэтому нетрудно убедиться в том, что его можно записать в виде

$$
(\psi, \varphi)_{\mathcal{H}}=\left(\psi_{2}, \varphi_{2}\right)+\left(\psi_{1}, \Lambda \varphi_{1}\right)
$$

ПРЕДЛОЖЕНИЕ 4.1. Для любъх $\zeta=(x, \eta(\nu)), \zeta^{\prime}=\left(x^{\prime}, \eta^{\prime}(\nu)\right)$ maкux, что $\nu \eta, \nu \eta^{\prime} \in L_{2}$, выполнено $\left(\zeta, \Lambda \zeta^{\prime}\right)=\left(\Lambda \zeta, \zeta^{\prime}\right)$.

ДокАЗАТЕЛЬство. Согласно замечанию 2.2 имеем $\widehat{a}_{l} / \nu \in L_{2}$ при всех $1 \leqslant$ $l \leqslant n$. Поскольку $\nu \eta \in L_{2}$, то

$$
\int \widehat{a}_{l} \eta d \nu=\int \frac{\widehat{a}_{l}}{\nu} \nu \eta d \nu<\infty .
$$

Также имеем $\int \widehat{a}_{l} \eta^{\prime} d \nu<\infty$. Поэтому оба выражения $\left(\zeta, \Lambda \zeta^{\prime}\right)$ и $\left(\Lambda \zeta, \zeta^{\prime}\right)$ определены корректно:

$$
\begin{aligned}
\left(\zeta, \Lambda \zeta^{\prime}\right) & =\left\langle x, B \bar{x}^{\prime}-\int \widehat{a} \bar{\eta}^{\prime} d \nu\right\rangle+\int \eta\left(\nu^{2} \bar{\eta}^{\prime}-\left\langle\widehat{a}, \bar{x}^{\prime}\right\rangle\right) d \nu \\
& =\left\langle B x-\int \widehat{a} \eta d \nu, \bar{x}^{\prime}\right\rangle+\int\left(\nu^{2} \eta-\langle\widehat{a}, x\rangle\right) \bar{\eta}^{\prime} d \nu=\left(\Lambda \zeta, \zeta^{\prime}\right)
\end{aligned}
$$

ПРЕДЛОЖЕНИЕ 4.2. Пусть $\psi=\left(\begin{array}{cc}x & \eta(\nu) \\ y & \zeta(\nu)\end{array}\right)$. Тогда $\psi \in \mathcal{H}$, если и толъко если

$$
\int\left(|\zeta|^{2}+\nu^{2}|\eta|^{2}\right) d \nu<\infty
$$


ДокАзАТЕльство. Импликация $\Rightarrow$ верна в силу очевидного следствия (3.3). Импликация $\Leftarrow$ верна в силу соотношения

$$
(\psi, \psi)_{\mathcal{H}} \leqslant\langle y, \bar{y}\rangle+c_{1}\langle x, \bar{x}\rangle+c_{2} \int\left(|\zeta|^{2}+\nu^{2}|\eta|^{2}\right) d \nu,
$$

где $c_{1}, c_{2}>0$ - постоянные. Обоснуем это соотношение.

Согласно (3.3) достаточно заметить, что выполняются следующие неравенства (в них использовано замечание $2.2\left(\widehat{a}_{l} / \nu \in L_{2}\right)$ и неравенство Коши-Буняковского):

$$
\begin{gathered}
\langle x, B \bar{x}\rangle \leqslant c_{3}\langle x, \bar{x}\rangle \\
\left|\left\langle x, \int \widehat{a} \bar{\eta} d \nu\right\rangle\right| \leqslant\langle x, \bar{x}\rangle^{1 / 2}\left\langle\int \widehat{a} \eta d \nu, \int \widehat{a} \bar{\eta} d \nu\right\rangle^{1 / 2} \\
\leqslant c_{4}\langle x, \bar{x}\rangle^{1 / 2}\left(\int \nu^{2}|\eta|^{2} d \nu\right)^{1 / 2} \leqslant \frac{c_{4}}{2}\left(\langle x, \bar{x}\rangle+\int \nu^{2}|\eta|^{2} d \nu\right),
\end{gathered}
$$

где $c_{3}=\|B\|, c_{4}=\left(\sum_{l=1}^{n} \int \frac{\widehat{a}_{l}^{2}}{\nu^{2}} d \nu\right)^{1 / 2}$. Положим $c_{1}=c_{3}+c_{4}, c_{2}=1+c_{4}$.

СлЕДСТвиЕ 4.1. Вектор $\psi \in \mathcal{H}$ лежит в $D(\Omega)$ - области определения оператора $\Omega$ тогда и только тогда, когда

$$
\nu^{2} \eta-\langle\widehat{a}, x\rangle, \quad \nu \zeta \in L_{2} .
$$

ДокАзАтЕльство. Согласно (3.6) оператор $\Omega$ действует следующим образом:

$$
\Omega \psi=i\left(\begin{array}{cc}
-y & -\zeta \\
B x-\int \widehat{a} \eta d \nu & \nu^{2} \eta-\langle\widehat{a}, x\rangle
\end{array}\right) .
$$

По определению имеем $\psi \in D(\Omega) \Leftrightarrow \Omega \psi \in \mathcal{H}$. Для завершения доказательства остается применить предложение 4.2 к формуле (4.4).

ДоКАЗАТЕЛЬСТво ПРЕДЛОЖЕНИЯ 3.1. Неограниченность вытекает из следствия 4.1.

Самосопряженность. Согласно [10] сопряженный оператор $\Omega^{*}$ к неограниченному оператору $\Omega$ со всюду плотной областью определения задается следующим образом ${ }^{6}$ :

$$
\varphi \in D\left(\Omega^{*}\right) \quad \Leftrightarrow \quad(\Omega \psi, \varphi)_{\mathcal{H}}=\left(\psi, \varphi^{*}\right)
$$

для любого $\psi \in D(\Omega)$ и некоторого $\varphi^{*} \in \mathcal{H}$. При этом оператор $\Omega^{*}$ определяется равенством $\Omega^{*} \varphi=\varphi^{*}$.

Оператор $\Omega$ называется самосопряженнъм, когда $\Omega^{*}=\Omega$ (кроме того, должны совпадать их области определения). Поэтому для доказательства предложения достаточно проверить три условия:

(i) область определения $D(\Omega)$ плотна в $\mathcal{H}$;

(ii) для любых $\psi, \varphi \in D(\Omega)$ выполнено $(\Omega \psi, \varphi)_{\mathcal{H}}=(\psi, \Omega \varphi)_{\mathcal{H}}$;

\footnotetext{
${ }^{6}$ Плотность области определения $\Omega$ мы покажем чуть ниже.
} 
(iii) если для любого $\psi \in D(\Omega)$ и некоторых $\varphi, \varphi^{*} \in \mathcal{H}$ выполнено

$$
(\Omega \psi, \varphi)_{\mathcal{H}}=\left(\psi, \varphi^{*}\right)_{\mathcal{H}},
$$

то $\varphi \in D(\Omega)$.

Условие (ii) обеспечивает вложение $D(\Omega) \subset D\left(\Omega^{*}\right)$ и равенство $\Omega=\Omega^{*}$ на $D(\Omega)$, а условие (iii) - обратное вложение $D\left(\Omega^{*}\right) \subset D(\Omega)$.

(i) Укажем семейство векторов $\psi=\left(\begin{array}{ll}x & \eta(\nu) \\ y & \zeta(\nu)\end{array}\right), \psi \in D(\Omega)$, всюду плотное в $\mathcal{H}$. Построим его следующим образом.

Будем говорить, что $\psi \in \Psi_{N}, N \in \mathbb{N}$, если компоненты $\psi$ удовлетворяют:
1) $\zeta \in L_{2}$;
2) $\left.\zeta\right|_{|\nu|>N}=0$
3) $\nu \eta \in L_{2}(-N, N)$;
4) $\left.\eta\right|_{|\nu|>N}=\left.\left\langle\widehat{a} \nu^{-2}, x\right\rangle\right|_{|\nu|>N}$

Покажем, что $\Psi_{N} \subset \mathcal{H}$. Действительно, согласно замечанию 2.2 имеем $\widehat{a}_{l} / \nu \in L_{2}$, поэтому из 3$\left.), 4\right)$ следует $\nu \eta \in L_{2}$. С учетом 1$)$ из предложения 4.2 следует, что $\Psi_{N} \subset \mathcal{H}$.

Искомое семейство получим как $\Psi=\bigcup_{N \in \mathbb{N}} \Psi_{N} \subset \mathcal{H}$.

Покажем, что $\Psi \subset D(\Omega)$. Для этого достаточно проверить условия следствия 4.1. Для выбранного семейства они выполнены, так как из условий 1), 2) следует $\nu \zeta \in L_{2} ;$ из условия 4) следует $\left.\nu^{2} \eta\right|_{|\nu|>N}=\left.\langle\widehat{a}, x\rangle\right|_{|\nu|>N}$. Поэтому из 3$), 4)$ и $\widehat{a}_{l} / \nu \in L_{2}$ следует $\left(\nu^{2} \eta-\langle\widehat{a}, x\rangle\right) \in L_{2}$.

Условия 1)-4) налагают ограничения только на "хвосты функций”. Согласно определению скалярного произведения (3.3) их норма мала. Поэтому, очевидно, $\Psi$ всюду плотно в $\mathcal{H}$.

(ii) Пусть $\psi=\left(\begin{array}{ll}x^{\psi} & \eta^{\psi} \\ y^{\psi} & \zeta^{\psi}\end{array}\right), \varphi=\left(\begin{array}{ll}x^{\varphi} & \eta^{\varphi} \\ y^{\varphi} & \zeta^{\varphi}\end{array}\right), \psi, \varphi \in D(\Omega)$. Согласно предложению 4.2 из $\psi \in \mathcal{H}$ следует $\nu \eta^{\psi} \in L_{2}$. Согласно следствию 4.1 из $\varphi \in D(\Omega)$ вытекает $\nu \zeta^{\varphi} \in L_{2}$. Поэтому из предложения 4.1 следует, что $\left(\Lambda \psi_{1}, \varphi_{2}\right)=\left(\psi_{1}, \Lambda \varphi_{2}\right)$. Согласно (4.1) и определению оператора $\Omega,(3.6)$, имеем

$$
(\Omega \psi, \varphi)_{\mathcal{H}}=\left(i \Lambda \psi_{1}, \varphi_{2}\right)+\left(-i \psi_{2}, \Lambda \varphi_{1}\right)=\left(\psi_{2}, i \Lambda \varphi_{1}\right)+\left(\psi_{1},-i \Lambda \varphi_{2}\right)=(\psi, \Omega \varphi)_{\mathcal{H}} .
$$

(iii) Пусть для некоторых $\varphi, \varphi^{*} \in \mathcal{H}$ выполнено (4.5). Используя (3.6) и (4.1), перепишем (4.5) следующим образом:

$$
\left(i \Lambda \psi_{1}, \varphi_{2}\right)+\left(-i \psi_{2}, \Lambda \varphi_{1}\right)=\left(\psi_{2}, \varphi_{2}^{*}\right)+\left(\psi_{1}, \Lambda \varphi_{1}^{*}\right) .
$$

Поскольку $\psi \in D(\Omega)$ произвольно, из (4.6) следует, что:
a) $\left(i \Lambda \psi_{1}, \varphi_{2}\right)=\left(\psi_{1}, \Lambda \varphi_{1}^{*}\right)$
b) $\left(-i \psi_{2}, \Lambda \varphi_{1}\right)=\left(\psi_{2}, \varphi_{2}^{*}\right)$.

Так как $\psi, \varphi^{*} \in \mathcal{H}$, то согласно предложению 4.2 выполнены условия предложения 4.1: $\nu \eta^{\psi}, \nu \eta^{\varphi^{*}} \in L_{2}$. Поэтому, применяя предложение 4.1 к правой части равенства а), его можно переписать в виде

$$
\left.\mathrm{a}^{\prime}\right)\left(i \Lambda \psi_{1}, \varphi_{2}\right)=\left(\Lambda \psi_{1}, \varphi_{1}^{*}\right) .
$$

Поскольку $D(\Omega)$ всюду плотно, из а $\left.{ }^{\prime}\right)$, b) следуют равенства:

$$
\text { c) }-i \varphi_{2}=\varphi_{1}^{*} ; \quad \text { d) } i \Lambda \varphi_{1}=\varphi_{2}^{*} \text {. }
$$


Используя определение $\Lambda,(3.6)$, запишем в с) и d) равенство бесконечномерных компонент:

$$
\left.\left.\mathrm{c}^{\prime}\right)-i \zeta^{\varphi}=\eta^{\varphi^{*}} ; \quad \mathrm{d}^{\prime}\right) i\left(\nu^{2} \eta^{\varphi}-\left\langle\widehat{a}, x^{\varphi}\right\rangle\right)=\zeta^{\varphi^{*}} .
$$

Так как $\varphi^{*} \in \mathcal{H}$, согласно предложению 4.2 имеем

$$
\left.\left.\mathrm{c}^{\prime}\right) \quad \Rightarrow \quad \nu \zeta^{\varphi} \in L_{2}, \quad \mathrm{~d}^{\prime}\right) \quad \Rightarrow \quad \nu^{2} \eta^{\varphi}-\left\langle\widehat{a}, x^{\varphi}\right\rangle \in L_{2}
$$

Поэтому согласно следствию 4.1 выполнено $\varphi \in D(\Omega)$.

ДокАЗАТЕЛЬСТво ЛЕммы 3.1 основано на следующих утверждениях.

ПрЕДЛОЖЕНИЕ 4.3. Число $\lambda \in\left(-\nu_{0}, \nu_{0}\right)$ является собственным значением оператора $\Omega$ тогда и только тогда, когда

$$
\operatorname{det}\left(B-\lambda^{2} I-Q\left(\lambda^{2}\right)\right)=0
$$

где $I$ - единичная матрица размера $n \times n, a Q\left(\lambda^{2}\right)-$ матрища, состоящая из элементов $q_{i j}=\int \frac{\widehat{a}_{i}(\nu) \widehat{a}_{j}(\nu)}{\nu^{2}-\lambda^{2}} d \nu$.

ДокАзАтельство. Сначала заметим, что интегралы $\int \frac{\widehat{a}_{i} \widehat{a}_{j}}{\nu^{2}-\lambda^{2}} d \nu$, фигурирующие в определении семейства матриц $Q\left(\lambda^{2}\right)$, сходятся. Действительно, $\lambda \in\left(-\nu_{0}, \nu_{0}\right)$, и согласно Н6,1) имеем $\left.\widehat{a}_{l}\right|_{\left(-\nu_{0}, \nu_{0}\right)}=0$. Остается заметить, что согласно замечанию 2.2 выполнено $\widehat{a}_{l} / \nu \in L_{2}$.

Пусть $\lambda$ - собственное значение оператора $\Omega$. Тогда существует $\psi=\left(\begin{array}{c}\psi_{1} \\ \psi_{2}\end{array}\right)=$ $\left(\begin{array}{ll}x & \eta \\ y & \zeta\end{array}\right) \neq 0$ такое, что $(\Omega-\lambda \mathbb{I}) \psi=0$, где $\mathbb{I}-$ единичный оператор на $\mathcal{H}$. Согласно определению оператора (3.6) имеем

$$
\left\{\begin{array} { l } 
{ - \lambda \psi _ { 1 } - i \psi _ { 2 } = 0 } \\
{ i \Lambda \psi _ { 1 } - \lambda \psi _ { 2 } = 0 }
\end{array} \quad \Leftrightarrow \quad \left\{\begin{array}{l}
\psi_{2}=i \lambda \psi_{1} \\
\left(\Lambda-\lambda^{2}\right) \psi_{1}=0 .
\end{array}\right.\right.
$$

Перепишем соотношение $\left(\Lambda-\lambda^{2}\right) \psi_{1}=0$ более детально:

$$
\left(B-\lambda^{2} E\right) x-\int \widehat{a}(\nu) \eta(\nu) d \nu=0, \quad-\langle\widehat{a}(\nu), x\rangle+\left(\nu^{2}-\lambda^{2}\right) \eta(\nu)=0 .
$$

Выразим $\eta$ из последнего уравнения:

$$
\eta(\nu)=\frac{\langle\widehat{a}(\nu), x\rangle}{\nu^{2}-\lambda^{2}} .
$$

Подставляя (4.9) в (4.8), получим

$$
\left(B-\lambda^{2} E-Q\left(\lambda^{2}\right)\right) x=0 .
$$

Поскольку $\psi \neq 0$, то $x \neq 0$. Поэтому $\operatorname{det}\left(B-\lambda^{2} E-Q\left(\lambda^{2}\right)\right)=0$.

Остается показать, что полученный собственный вектор $\psi$ принадлежит $\mathcal{H}$. Для этого покажем, что выполнены условия предложения 4.2. Согласно H6, 1) имеем $\left.\widehat{a}\right|_{\left(-\nu_{0}, \nu_{0}\right)}=0$. Вне этого интервала знаменатель в (4.9) не обращается в нуль. Остальное нетрудно получить, используя замечание 2.2 , согласно которому $\widehat{a}_{l} / \nu \in L_{2}$. Предложение доказано. 
Обозначим

$$
V(\lambda)=B-\lambda I-Q(\lambda)
$$

Таким образом, согласно предложению 4.3 на промежутке $\left(-\nu_{0}, \nu_{0}\right)$ дискретный спектр оператора $\Omega$ состоит из корней уравнения $\operatorname{det} V\left(\lambda^{2}\right)=0$.

УтвеРЖДЕНИЕ 4.1. Число корней уравнения $\operatorname{det} V(\lambda)=0$, лежащих на промежутке $\left[0, \nu_{0}^{2}\right)$, совпадает с пределом $\lim _{\nu / \nu_{0}} \lambda_{V\left(\nu^{2}\right)}^{-}$, где $\lambda_{V\left(\nu^{2}\right)}^{-}$- число отрицательных собственных значений матрицы $V\left(\nu^{2}\right)$. Нуль не является корнем данного уравнения.

ДокАзАТЕЛЬство. Матрицы семейства $V(\lambda)$ симметричны, поэтому каждая из них имеет ровно $n$ действительных собственных значений $\mu_{k}$. Функции $\mu_{k}(\lambda)$ непрерывны для каждого $k=1, \ldots, n$. Доказательство утверждения основано на свойстве монотонности функций $\mu_{k}(\lambda)$.

ПРЕДЛОЖЕНИЕ 4.4. Функиии $\mu_{k}(\lambda)$ монотонно убъвают на промежутке $\left[0, \nu_{0}^{2}\right)$ для каждого $k=1, \ldots, n$.

Доказательство предложения 4.4 дается после окончания доказательства леммы.

Далее, $\operatorname{det} V(\lambda)=0$ тогда и только тогда, когда какое-либо из собственных значений $\mu_{k}(\lambda)$ матрицы $V(\lambda)$ обратилось в нуль.

Нетрудно убедиться в том, что $V(0)=B-K$, где матрица $K$ определена в Н3. Согласно Н3 матрица $B-K$ положительно определена, поэтому $\mu_{k}(0)>0$ для каждого $k=1, \ldots, n$. Согласно предложению 4.4 функции $\mu_{k}(\lambda)$ монотонно убывают по $\lambda$. Поэтому суммарное число корней уравнений $\mu_{k}(\lambda)=0$, $k=1, \ldots, n$, на промежутке $\left[0, \nu_{0}^{2}\right)$ равно количеству $\mu_{k}(\lambda)$, ставших отрицательными к моменту $\lambda=\nu_{0}^{2}$, что и завершает доказательство утверждения ${ }^{7}$.

СледСтвиЕ 4.2. На промежутке $\left[0, \nu_{0}^{2}\right)$ уравнение $\operatorname{det} V(\lambda)=0$ имеет не больше $n$ корней.

ПреДЛОЖеНИЕ 4.5. На промежутке $\left(-\nu_{0}, \nu_{0}\right)$ оператор $\Omega$ имеет толъко дискретныи спектр.

ДокАзАтельство. Согласно предложению 4.3 из равенства $\operatorname{det} V\left(\lambda^{2}\right)=0$, $\lambda \in\left(-\nu_{0}, \nu_{0}\right)$, следует, что $\lambda-$ собственное значение оператора $\Omega$. Покажем, что для любого $\lambda \in\left(-\nu_{0}, \nu_{0}\right), \operatorname{det} V\left(\lambda^{2}\right) \neq 0$, оператор $\Omega-\lambda \mathbb{I}$ обратим.

Действительно, рассмотрим уравнение

$$
(\Omega-\lambda \mathbb{I}) \psi=\varphi, \quad \text { где } \quad \psi=\left(\begin{array}{l}
\psi_{1} \\
\psi_{2}
\end{array}\right)=\left(\begin{array}{ll}
x^{\psi} & \eta^{\psi} \\
y^{\psi} & \zeta^{\psi}
\end{array}\right), \quad \varphi=\left(\begin{array}{l}
\varphi_{1} \\
\varphi_{2}
\end{array}\right)=\left(\begin{array}{ll}
x^{\varphi} & \eta^{\varphi} \\
y^{\varphi} & \zeta^{\varphi}
\end{array}\right) .
$$

Согласно определению оператора (3.6) имеем

$$
\left\{\begin{array} { l } 
{ - \lambda \psi _ { 1 } - i \psi _ { 2 } = \varphi _ { 1 } } \\
{ i \Lambda \psi _ { 1 } - \lambda \psi _ { 2 } = \varphi _ { 2 } }
\end{array} \Leftrightarrow \left\{\begin{array}{l}
\psi_{2}=i \lambda \psi_{1}+i \varphi_{1} \\
\left(\Lambda-\lambda^{2}\right) \psi_{1}=\lambda \varphi_{1}-i \varphi_{2} .
\end{array}\right.\right.
$$

\footnotetext{
${ }^{7}$ Предел нужен, так как интегралы в определении матрицы $Q(\lambda)$, вообще говоря, могут расходиться в точке $\lambda=\nu_{0}^{2}$.
} 
Перепишем соотношение $\left(\Lambda-\lambda^{2}\right) \psi_{1}=\lambda \varphi_{1}-i \varphi_{2}$ более подробно:

$$
\begin{gathered}
\left(B-\lambda^{2} E\right) x^{\psi}-\int \widehat{a}(\nu) \eta^{\psi}(\nu) d \nu=\lambda x^{\varphi}-i y^{\varphi}, \\
-\left\langle\widehat{a}(\nu), x^{\psi}\right\rangle+\left(\nu^{2}-\lambda^{2}\right) \eta^{\psi}(\nu)=\lambda \eta^{\varphi}-i \zeta^{\varphi} .
\end{gathered}
$$

Выразим $\eta^{\psi}$ из последнего уравнения в (4.11):

$$
\eta^{\psi}(\nu)=\frac{\lambda \eta^{\varphi}-i \zeta^{\varphi}+\left\langle\widehat{a}(\nu), x^{\psi}\right\rangle}{\nu^{2}-\lambda^{2}} .
$$

Подставим (4.12) в первое уравнение из (4.11). Получим уравнение $V\left(\lambda^{2}\right) x=z$, где $z \in \mathbb{C}^{n}$. Поскольку $\operatorname{det} V\left(\lambda^{2}\right) \neq 0$, уравнение имеет решение.

Остается показать, что полученное решение $\psi$ принадлежит $\mathcal{H}$. Для этого нужно проверить выполнение условий предложения 4.2. Согласно Н6, 1) имеем $\left.\widehat{a}\right|_{\left(-\nu_{0}, \nu_{0}\right)}=0$. Поэтому согласно $(3.8)$ на интервале $\left(-\nu_{0}, \nu_{0}\right)$, т. е. там, где знаменатель в (4.12) может обращаться в нуль, числитель тождественно равен нулю. Остальное легко получить, используя замечание 2.2 , согласно которому $\widehat{a}_{l} / \nu \in L_{2}$. Предложение доказано.

Таким образом, мы показали, что оператор $\Omega$ на промежутке $\left(-\nu_{0}, \nu_{0}\right)$ имеет только дискретный спектр, состоящий из корней уравнения $\operatorname{det} V\left(\lambda^{2}\right)=0$, причем корней не более $n$ и нуль не является корнем. Доказательство леммы закончено.

ДОКАЗАТЕЛЬСТво ПРЕДЛОЖЕНИЯ 4.4. Сначала покажем, что семейство $V(\lambda)$ монотонно убывает, т. е. квадратичные формы $V\left(\lambda_{1}\right)-V\left(\lambda_{2}\right)$ положительно определены для любых $0 \leqslant \lambda_{1}<\lambda_{2}<\nu_{0}^{2}$. Действительно,

$$
V\left(\lambda_{1}\right)-V\left(\lambda_{2}\right)=\left(\lambda_{2}-\lambda_{1}\right) I+Q\left(\lambda_{2}\right)-Q\left(\lambda_{1}\right) .
$$

Матрица $\left(\lambda_{2}-\lambda_{1}\right) I$ определена положительно. Элементы матрицы $Q\left(\lambda_{2}\right)-$ $Q\left(\lambda_{1}\right)$ имеют вид

$$
q_{i j}\left(\lambda_{2}\right)-q_{i j}\left(\lambda_{1}\right)=\int \widehat{a}_{i} \widehat{a}_{j}\left(\frac{1}{\nu^{2}-\lambda_{2}}-\frac{1}{\nu^{2}-\lambda_{1}}\right) d \nu,
$$

причем $\frac{1}{\nu^{2}-\lambda_{2}}-\frac{1}{\nu^{2}-\lambda_{1}}>0$ при $0 \leqslant \lambda_{1}<\lambda_{2}<\nu_{0}^{2}$. Значит, $Q\left(\lambda_{2}\right)-Q\left(\lambda_{1}\right)$ является матрицей Грамма системы векторов $z_{i}(\nu)=\widehat{a}_{i}(\nu) \sqrt{\frac{1}{\nu^{2}-\lambda_{2}}-\frac{1}{\nu^{2}-\lambda_{1}}}, i=1, \ldots, n$, и определена неотрицательно.

В $[11, \S 17]$ доказана следующая формула. Пусть $V$ - произвольная симметричная матрица. Упорядочим ее собственные значения по возрастанию: $\mu_{1} \leqslant \mu_{2} \leqslant \cdots \leqslant \mu_{n}$. Тогда для любого $k=1, \ldots, n$

$$
\mu_{k}=\max _{R_{k}} \min _{x \in R_{k}} \frac{\langle V x, x\rangle}{\langle x, x\rangle},
$$

где $R_{k}$ - всевозможные пространства размерности $n-k+1$.

Поскольку матрица $V\left(\lambda_{1}\right)-V\left(\lambda_{2}\right)$ положительно определена, имеем

$$
\frac{\left\langle V\left(\lambda_{2}\right) x, x\right\rangle}{\langle x, x\rangle}<\frac{\left\langle V\left(\lambda_{1}\right) x, x\right\rangle}{\langle x, x\rangle} .
$$

Согласно (4.13) получаем $\mu_{k}\left(\lambda_{2}\right)<\mu_{k}\left(\lambda_{1}\right)$. 


\section{§5. Примеры}

5.1. Термостат Клейна-Гордона. Предположения Н6,2)-4) являются условиями общего положения, а Н6,1) может показаться несколько необычным. В этом пункте мы приводим естественный пример системы, для которой выполнено условие Н6, 1).

Рассмотрим уравнения

$$
\begin{gathered}
\ddot{y}+B y=\int f u d q, \\
u_{t t}=u_{q q}-\nu_{0}^{2} u+\langle f, y\rangle,
\end{gathered}
$$

где $u=u(q, t), f=f(q)$ - действительная вектор-функция, преобразование Фурье $\hat{f}$ которой также действительно, $\nu_{0}-$ постоянная, $y=y(t) \in \mathbb{R}^{n}$, точки сверху, как обычно, обозначают дифференцирование по времени. Однородная часть соотношения (5.2) представляет собой хорошо известное уравнение Клейна-Гордона.

Применим к уравнению (5.2) преобразование Фурье $\eta(s, t)=\int e^{-i q s} u(q, t) d q$. Учтем, что $\int f u d q=\frac{1}{2 \pi} \int \hat{f} \eta d s$. Обозначим $g=\frac{\hat{f}}{\sqrt{2 \pi}}, x=\sqrt{2 \pi} y$. Получим следующую систему:

$$
\begin{gathered}
\ddot{x}+B x=\int g \eta d s, \\
\ddot{\eta}+\left(s^{2}+\nu_{0}^{2}\right) \eta=\langle g, x\rangle .
\end{gathered}
$$

Соответствующий лагранжиан имеет вид $\mathcal{L}=\mathcal{L}_{T}+\mathcal{L}_{0}-V_{\text {int }}$, где

$\mathcal{L}_{T}=\frac{1}{2} \int\left(\dot{\eta}^{2}-\left(s^{2}+\nu_{0}^{2}\right) \eta^{2}\right) d s, \quad \mathcal{L}_{0}=\frac{1}{2}\langle\dot{x}, \dot{x}\rangle-\frac{1}{2}\langle x, B x\rangle, \quad V_{\text {int }}=-\left\langle x, \int g \eta d s\right\rangle$.

Положим $\nu^{2}=\nu_{0}^{2}+s^{2}, \nu s \geqslant 0$. Выберем произвольную функцию $\rho(\nu)>0$. Учитывая соотношение $s d s=\nu d \nu$, получим

$$
\mathcal{L}_{T}=\frac{1}{2} \int \rho(\nu)\left(\dot{\xi}^{2}-\nu^{2} \xi^{2}\right) d \nu, \quad V_{\mathrm{int}}=-\left\langle x, \int \kappa \xi d \nu\right\rangle,
$$

где

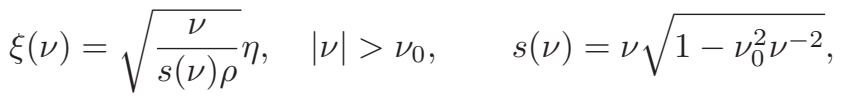

$$
\begin{aligned}
& \kappa(\nu)=\left\{\begin{array}{lll}
0, & \text { если } & |\nu| \leqslant \nu_{0}, \\
\sqrt{\frac{\nu \rho}{s(\nu)}} g(s(\nu)), & \text { если } & |\nu|>\nu_{0} .
\end{array}\right.
\end{aligned}
$$

Заметим, что мы получили систему с лагранжианом, в точности совпадающим с лагранжианом системы (1.4), (1.5).

Имеет место следующее очевидное

ПредлОжЕнИЕ 5.1. Пусть $g(s)$ - дифферениируемая функиия и $g(0)=0$. Тогда функиии $\widehat{a}_{l}, \widehat{w}_{l m}$, определенные в (2.2), непрерывны и выполнены равенcmsa $\left.\widehat{a}_{l}\right|_{\left(-\nu_{0}, \nu_{0}\right)}=\left.\widehat{w}_{l m}\right|_{\left(-\nu_{0}, \nu_{0}\right)}=0$.

Заметим, что в этом случае интервал $\left(-\nu_{0}, \nu_{0}\right)$ удовлетворяет условию H6, 1$)$. 
5.2. Общий случай линейного взаимодействия. Здесь более общая линейная система будет сведена к системе, рассмотренной в 1 .

Введем некоторые обозначения. Зафиксируем $-\infty \leqslant p<q \leqslant+\infty$. Для почти всюду положительной функции $\varphi(s):(p, q) \rightarrow \mathbb{R}$ обозначим

$$
L_{2}^{\varphi}=\left\{f(s) \mid \int_{p}^{q} \varphi f^{2} d s<\infty\right\} .
$$

Рассмотрим систему, состоящую из конечномерной и бесконечномерной частей, положение которых описывается координатами $u(t) \in \mathbb{R}^{n}$ и $f(s, t)$ : $(p, q) \times \mathbb{R} \rightarrow \mathbb{R}$ соответственно, где $s \in(p, q)$ параметризует бесконечномерную часть. Пусть кинетическая и потенциальная энергии имеют следующий вид:

$$
\begin{aligned}
T & =\frac{1}{2}\langle\dot{u}, A \dot{u}\rangle-\left\langle\dot{u}, \int_{p}^{q} \alpha(s) \dot{f}(s) d s\right\rangle+\frac{1}{2} \int_{p}^{q} a(s) \dot{f}^{2}(s) d s \\
V & =\frac{1}{2}\langle u, H u\rangle-\left\langle u, \int_{p}^{q} \gamma(s) f(s) d s\right\rangle+\frac{1}{2} \int_{p}^{q} h(s) f^{2}(s) d s,
\end{aligned}
$$

где $a(s)$ и $h(s)$ - некоторые почти всюду положительные функции, $\alpha=$ $\left(\alpha_{1}, \ldots, \alpha_{n}\right), \gamma=\left(\gamma_{1}, \ldots, \gamma_{n}\right)$ такие, что $\frac{\alpha_{i}}{\sqrt{a}}, \frac{\gamma_{i}}{\sqrt{h}} \in L_{2}(p, q), A$ и $H$ - симметрические положительно определенные матрицы размера $n \times n$. Все функции мы считаем действительными.

Нетрудно видеть, что кинетическая и потенциальная энергии корректно определены соответственно на векторах $(\dot{u}, \dot{f}),(u, f), \dot{u}, u \in \mathbb{R}^{n}, \dot{f} \in L_{2}^{a}, f \in L_{2}^{h}$.

Кинетическая энергия предполагается положительно определенной, т. е. положительной для любого ненулевого вектора $(\dot{u}, \dot{f}), \dot{f} \in L_{2}^{a}$.

Уравнения движения системы с лагранжианом $L=T-V$ имеют вид

$$
A \ddot{u}-\int_{p}^{q} \alpha \dot{f} d s=-H u+\int_{p}^{q} \gamma f d s, \quad a \ddot{f}-\langle\dot{u}, \alpha\rangle=-h f+\langle u, \gamma\rangle .
$$

УтВеРЖДЕНИЕ 5.1. Пусть выполнены следующие предположения:

1) кинетическая энергия положительно определена;

2) $\frac{\alpha_{i}}{\sqrt{a}}, \frac{\gamma_{i}}{\sqrt{h}}, \frac{\sqrt{h} \alpha_{i}}{a} \in L_{2}(p, q)$;

3) функиия $\frac{h(s)}{a(s)}$ дифференцируема, $\left(\frac{h(s)}{a(s)}\right)^{\prime}>0$, inf $\frac{h(s)}{a(s)}=\nu_{0}^{2}$ для некоторой постоянной $\nu_{0} \geqslant 0 u \frac{h(s)}{a(s)} \rightarrow \infty$ nри $s \rightarrow q$;

4) для функиии $\rho=\sqrt{\frac{a}{h}}\left(\frac{h}{a}\right)^{\prime}$ выполнено $\lim _{s \rightarrow p} \rho<\infty$.

Тогда система (5.8) при помощи замены переменных сводится $к$ системе вида (1.4), (1.5).

ДокАзАтельство. 1. Сначала диагонализуем кинетическую энергию. Для этого перепишем ее в следующем виде:

$$
2 T=\int_{p}^{q}\left(\sqrt{a} \dot{f}-\left\langle\dot{u}, \frac{\alpha}{\sqrt{a}}\right\rangle\right)^{2} d s+\langle\dot{u}, \tilde{A} \dot{u}\rangle
$$

где $\tilde{A}$ - матрица с элементами $a_{i j}-\int_{p}^{q} \frac{\alpha_{i} \alpha_{j}}{a} d s$.

Далее нам будет нужно следующее. 
ПредложениЕ 5.2. Матрица Ӓ положительно определена.

ДоказАтельство. В (5.9) выберем произвольно $\dot{u} \neq 0$. Возьмем $\dot{f}=\left\langle\dot{u}, \frac{\alpha}{a}\right\rangle$. Согласно условию 2) имеем $\dot{f} \in L_{2}^{a}(p, q)$, поэтому кинетическая энергия определена корректно. Поскольку $\dot{u} \neq 0$, согласно условию 1$)$ имеем $T>0$. В выражении (5.9) первое слагаемое обратится в нуль. Отсюда следует искомое предложение.

Сделаем замену $(u, f) \mapsto(x, \eta), x=\tilde{A}^{1 / 2} u, \eta=\sqrt{a} f-\left\langle u, \frac{\alpha}{\sqrt{a}}\right\rangle$. Заметим, что если $f \in L_{2}^{h}$, то $\eta \in L_{2}^{h / a}$. Тогда кинетическая энергия примет вид

$$
2 T=\int_{p}^{q} \dot{\eta}^{2} d s+\langle\dot{x}, \dot{x}\rangle .
$$

Прямым вычислением можно убедиться в том, что потенциальная энергия примет вид

$$
2 V=\langle x, B x\rangle-\left\langle x, \int_{p}^{q} \kappa \eta d s\right\rangle+\int_{p}^{q} \frac{h(s)}{a(s)} \eta^{2}(s) d s,
$$

где $B=\tilde{A}^{-1 / 2} \widetilde{H} \tilde{A}^{-1 / 2}, \widetilde{H}$ - матрица с элементами

$$
h_{i j}+\int_{p}^{q} \frac{h \alpha_{i} \alpha_{j}}{a^{2}} d s-\int_{p}^{q} \frac{\gamma_{i} \alpha_{j}+\gamma_{j} \alpha_{i}}{a} d s,
$$

$\kappa=\left(\kappa_{1}, \ldots, \kappa_{n}\right), \kappa=\widetilde{\gamma} \tilde{A}^{-1 / 2}, \widetilde{\gamma}=\left(\widetilde{\gamma}_{1}, \ldots, \widetilde{\gamma}_{n}\right), \widetilde{\gamma}_{i}=\frac{h \alpha_{i}}{a \sqrt{a}}-\frac{\gamma_{i}}{\sqrt{a}}$.

Из условия 2) следует, что для любого $i=1, \ldots, n$ интегралы $\int_{p}^{q} \kappa_{i} \eta d s$ сходятся при $\eta \in L_{2}^{h / a}$, а также сходятся все интегралы в определении $\widetilde{H}$. Таким образом, система (5.8) принимает вид

$$
\ddot{x}+B x=\int_{p}^{q} \kappa \eta d s, \quad \ddot{\eta}+\frac{h}{a} \eta=\langle\kappa, x\rangle .
$$

2. Согласно условию 3) корректно определена замена параметра: $s \mapsto \nu=$ $\sqrt{\frac{h}{a}}$, где $\nu$ определено на промежутке $\left[\nu_{0}, \infty\right), \nu_{0} \geqslant 0$. Тогда $d s=\frac{2 d \nu}{\rho}$, где $\rho=\frac{1}{\nu}\left(\frac{h}{a}\right)^{\prime}$. Продолжим функции $\kappa_{i}$ нулем на промежуток $\left(0, \nu_{0}\right)$, а функцию $\rho$ на этом промежутке выберем произвольной и отличной от нуля. Обозначим $\xi=\frac{\eta}{\rho}$. Система (5.11) примет вид

$$
\ddot{x}+B x=2 \int_{0}^{\infty} \kappa \xi d \nu, \quad \rho\left(\ddot{\xi}+\nu^{2} \xi\right)=\langle\kappa, x\rangle .
$$

Продолжим функции $\kappa_{i}$ четным образом и рассмотрим систему

$$
\ddot{x}+B x=\int \kappa \xi d \nu, \quad \rho\left(\ddot{\xi}+\nu^{2} \xi\right)=\langle\kappa, x\rangle .
$$

Нетрудно видеть, что каждому решению системы (5.12) однозначно соответствует решение системы (5.13) с четными начальными условиями. Это завершает доказательство утверждения. 


\section{§6. Окончание доказательства теоремы 2.1}

ПРЕДЛОЖЕНИЕ 6.1. Если энергия Е конечна, то функции $x_{l}(t)$ u $\dot{x}_{l}(t)$ ограничены для каждого $1 \leqslant l \leqslant n,\left|x_{l}\right| \leqslant c_{1}=c_{1}(E),\left|\dot{x}_{l}\right| \leqslant c_{2}=c_{2}(E)$.

ДокАЗАТЕЛЬСтво. Из соотношения (2.1) и условия Н3 следует, что если $x_{l}$ неограничены, то $\langle(B-K) x, x\rangle$ положительно и неограничено и $E=+\infty$. Если неограничены функции $\dot{x}_{l}$, то в $(2.1)$ неограничено $\langle\dot{x}, \dot{x}\rangle$. Предложение доказано.

Из ограниченности скоростей следует равномерная непрерывность функций $x_{l}(t)$, т. е. мы доказали свойство 1) из теоремы 2.1.

ПреДЛОжЕНИЕ 6.2. Если энергия $Е$ конечна, то $\left|\left\langle x, \int \kappa \xi d \nu\right\rangle\right| \leqslant c_{3}=c_{3}(E)$.

ДоказАтельство. Действительно, согласно неравенству Коши-Буняковского

$$
\left(\int \kappa_{s} \xi d \nu\right)^{2}=\left(\int \frac{\kappa_{s}}{\sqrt{\rho} \nu} \sqrt{\rho} \nu \xi d \nu\right)^{2} \leqslant k_{s s} \int \rho \nu^{2} \xi^{2} d \nu, \quad k_{s s}=\int \frac{\kappa_{s}^{2}}{\rho \nu^{2}} d \nu,
$$

поэтому

$$
\left\langle x, \int \kappa \xi d \nu\right\rangle^{2} \leqslant \sum_{l=1}^{n} x_{l}^{2} \sum_{s=1}^{n}\left(\int \kappa_{s} \xi d \nu\right)^{2} \leqslant\langle x, x\rangle \int \rho \nu^{2} \xi^{2} d \nu \sum_{s=1}^{n} k_{s s} .
$$

Согласно Н2 имеем $k_{s s}<\infty$. Из $(2.1)$ следует, что

$$
\int \rho \nu^{2} \xi^{2} d \nu \leqslant 2\left(E+\left\langle x, \int \kappa \xi d \nu\right\rangle\right)
$$

поэтому

$$
\left\langle x, \int \kappa \xi d \nu\right\rangle^{2} \leqslant 2\langle x, x\rangle \sum_{s=1}^{n} k_{s s}\left(E+\left\langle x, \int \kappa \xi d \nu\right\rangle\right)
$$

что равносильно

$$
\left(\left\langle x, \int \kappa \xi d \nu\right\rangle-\langle x, x\rangle \sum_{s=1}^{n} k_{s s}\right)^{2} \leqslant\langle x, x\rangle \sum_{s=1}^{n} k_{s s}\left(2 E+\langle x, x\rangle \sum_{s=1}^{n} k_{s s}\right) .
$$

С учетом предложения 6.1 получаем искомое утверждение.

ПРЕДЛОЖЕНИЕ 6.3. Если энергия Е конечна, то

$$
\mathcal{E}:=\int \rho\left(\dot{\xi}^{2}+\nu^{2} \xi^{2}\right) d \nu \leqslant c_{4}=c_{4}(E) .
$$

ДокАЗАТЕльство немедленно следует из (1.7) и предложений 6.1, 6.2. 


\section{§ 7. Доказательство теоремы 2.2}

Пусть $\chi_{M}$ - характеристическая функция множества $M \subset \mathbb{R}$. Если $M-$ интервал $\left(b_{1}, b_{2}\right) \subset \mathbb{R}$, то мы будем учитывать его ориентацию:

$$
\chi_{\left(b_{1}, b_{2}\right)}(t)=\left\{\begin{array}{lll}
1, & \text { если } & b_{1}<t<b_{2}, \\
-1, & \text { если } & b_{2}<t<b_{1}, \\
0, & \text { если } & \left(b_{1}-t\right)\left(t-b_{2}\right) \leqslant 0 .
\end{array}\right.
$$

Пусть $(x(t), \xi(\nu, t))$ - решение системы (1.4)-(1.6) с энергией

$$
E(x(t), \xi(\nu, t))<\infty .
$$

Для любого действительного $t$ положим

$$
x_{l}^{t}=\chi_{[0, t]} x_{l}(\tau), \quad \widehat{x}_{l}^{t}=\mathcal{F}\left(x_{l}^{t}\right), \quad 1 \leqslant l \leqslant n .
$$

Тогда $\widehat{x}_{l}^{t}$ - целые аналитические функции. Поскольку $x_{l}^{t}$ действительны, имеем

$$
\widehat{x}_{l}^{t}(\lambda)=\overline{\widehat{x}}_{l}^{t}(-\lambda) \text {. }
$$

Рассмотрим соответствующий вектор $\widehat{x}^{t}(\lambda) \in \widehat{\mathcal{C}}_{b}^{n}$.

Теперь рассмотрим уравнение $(1.5)$, считая, что $x(t)$ известно. С учетом соотношений (1.6) и (7.1) получим $\xi(\nu, t)=\xi_{1}(\nu, t)+\xi_{2}(\nu, t)$, где

$$
\begin{aligned}
\xi_{2}(\nu, t):= & \xi_{0}(\nu) \cos (\nu t)+\frac{1}{\nu} \dot{\xi}_{0}(\nu) \sin (\nu t)=\operatorname{Re}\left(\frac{1}{i \nu} \xi_{\bullet} e^{i \nu t}\right), \quad \xi_{\bullet}:=i \nu \xi_{0}+\dot{\xi}_{0} \\
\xi_{1}(\nu, t) & =\left\langle\frac{\kappa}{\rho \nu}, \int_{0}^{t} \sin (\nu(t-\tau)) x(\tau) d \tau\right\rangle \\
& =\left\langle\frac{\kappa}{2 i \rho \nu}, \int_{0}^{t}\left(e^{i \nu t-i \nu \tau}-e^{-i \nu t+i \nu \tau}\right) x(\tau) d \tau\right\rangle \\
& =\left\langle\frac{\kappa}{2 i \rho \nu},\left(e^{i \nu t} \widehat{x}^{t}(\nu)-e^{-i \nu t} \widehat{x}^{t}(-\nu)\right)\right\rangle=\operatorname{Re}\left\langle\frac{\kappa}{i \rho \nu}, \widehat{x}^{t}(\nu) e^{i \nu t}\right\rangle .
\end{aligned}
$$

Обозначим через $\|\cdot\|_{L_{2}}$ норму в $L_{2}:\|g\|_{L_{2}}^{2}=\int|g(\nu)|^{2} d \nu$ для любого $g: \mathbb{R} \rightarrow \mathbb{C}$.

7.1. Доказательство утверждения 2) теоремы 2.2. Обозначим

$$
\begin{gathered}
\widehat{\gamma}(\nu)=2 \pi \frac{\kappa \xi \bullet}{\nu}, \\
\mathcal{A}_{0}^{+}(t)=-\int_{t}^{\infty} W(\tau) x(t-\tau) d \tau, \quad \mathcal{A}_{0}^{-}(t)=\int_{-\infty}^{t} W(\tau) x(t-\tau) d \tau, \\
\gamma=\left(\gamma_{1}, \ldots, \gamma_{n}\right), \quad \mathcal{A}_{0}^{\star}=\left(\mathcal{A}_{01}^{\star}, \ldots, \mathcal{A}_{0 n}^{\star}\right), \quad \star \in\{+,-\} .
\end{gathered}
$$

ПреДЛОЖЕНИЕ 7.1. Для любого $1 \leqslant l \leqslant n$ имеем $\widehat{\gamma}_{l} \in L_{1}$.

ДокАЗАТЕЛЬСтво. Заметим, что

$$
\sqrt{\rho} \xi_{\bullet}, \frac{\kappa_{l}}{\sqrt{\rho} \nu} \in L_{2} .
$$

Действительно, первое включение следует из определения $\xi_{\bullet}=i \nu \xi_{0}+\dot{\xi}_{0}$ и условия Н4, а второе - из Н2. Поэтому $\frac{\kappa_{l} \xi_{\bullet}}{\nu} \in L_{1}$. 
ПРЕДЛОЖЕНИЕ 7.2. Для любого $1 \leqslant l \leqslant n$ имеем

$$
\lim _{t \rightarrow+\infty} \mathcal{A}_{0 l}^{+}(t)=\lim _{t \rightarrow-\infty} \mathcal{A}_{0 l}^{-}(t)=0 .
$$

Доказательство предложения 7.2 приведено в конце параграфа.

Лемма 7.1. Пусть $\gamma=\mathcal{F}^{-1}(\widehat{\gamma})$. Тогда

$$
\int \kappa \xi d \nu=\mathcal{F}^{-1}\left(\widehat{W}_{\diamond}^{ \pm} \widehat{x}\right)+\operatorname{Re}\left(\gamma(t)+\mathcal{A}_{0}^{ \pm}(t)\right) .
$$

СлЕДСтвиЕ 7.1. Выполняется утверждение 2) теоремъ 2.2:

$$
\int \kappa \xi d \nu=\mathcal{F}^{-1}\left(\widehat{W}_{\diamond}^{ \pm} \widehat{x}\right)+\vartheta^{ \pm}, \quad \vartheta^{ \pm} \in \mathcal{C}_{0}^{ \pm n}
$$

ДокАзАТЕЛЬСтво лЕммы 7.1. Сначала заметим, что согласно (7.3)

$$
\int \kappa \xi_{2} d \nu=\frac{1}{2 \pi} \operatorname{Re} \int e^{i \lambda t} \widehat{\gamma}(\lambda) d \lambda=\operatorname{Re} \gamma(t)
$$

Из (7.3) следует, что

$$
\begin{aligned}
\int \kappa \xi_{1} d \nu & =\operatorname{Re} \mathcal{A}(t), \\
\mathcal{A}(t):=\frac{1}{2 \pi} \int e^{i \nu t} \widehat{W}(\nu) \widehat{x}^{t}(\nu) d \nu & =\mathcal{F}^{-1}\left(\widehat{W} \widehat{x}^{t}\right)(t)=\left(W * x^{t}\right)(t),
\end{aligned}
$$

где $\left(W * x^{t}\right)(t)$ вычисляется по обычному правилу умножения матрицы на вектор, только вместо операции умножения делается свертка.

Согласно Н5 имеем $w_{l m} \in \mathcal{M}$. Поэтому $\mathcal{A}(t)=\mathcal{A}_{0}^{ \pm}(t)+\mathcal{A}_{1}^{ \pm}(t)$, где $\mathcal{A}_{0}^{ \pm}$определяются формулами из (7.4), и

$$
\mathcal{A}_{1}^{ \pm}(t)=\int \chi_{[0, \pm \infty)}(\tau) W(\tau) x(t-\tau) d \tau=\left(\chi_{[0, \pm \infty)} W\right) * x(t)
$$

Функции $x_{m}$ действительны, а функции $\widehat{w}_{l m}$ чисто мнимые. Поэтому $\bar{W}(\tau)=$ $-W(-\tau), \tau \in \mathbb{R}$. Следовательно,

$$
\operatorname{Re} \mathcal{A}_{1}^{ \pm}=\frac{1}{2}\left(\chi_{[0, \pm \infty)}(\tau) W(\tau)-\chi_{[0, \pm \infty)}(\tau) W(-\tau)\right) * x=\mathcal{F}^{-1}\left(\widehat{W}_{\diamond}^{ \pm} \widehat{x}\right),
$$

где $\widehat{W}_{\diamond}^{ \pm}-$матрицы, состоящие из элементов $\widehat{w}_{\diamond l m}^{ \pm}$,

$$
\widehat{w}_{\diamond l m}^{ \pm}=\frac{1}{2} \mathcal{F}\left(\chi_{[0, \pm \infty)}\left(w_{l m}(\tau)-w_{l m}(-\tau)\right)\right)=\frac{1}{4 \pi}\left(\widehat{\chi}_{[0, \pm \infty)} *\left(\widehat{w}_{l m}(\lambda)-\widehat{w}_{l m}(-\lambda)\right)\right) .
$$

Используя явную форму обобщенной функции [12]

$$
\widehat{\chi}_{[0, \pm \infty)}(\lambda)= \pm \pi \delta(\lambda)+\frac{1}{i} \text { v.p. } \frac{1}{\lambda},
$$

получаем (2.3).

ДокАЗАТЕЛЬСТво ЛЕммы 7.2. Имеем

$$
\left|\mathcal{A}_{0 l}^{ \pm}(t)\right|=\left|\sum_{m=1}^{n} x_{m} *\left(\chi_{[t, \pm \infty)} w_{l m}\right)(t)\right| \leqslant \sum_{m=1}^{n}\left\|\chi_{[t, \pm \infty)} w_{l m}\right\|_{\mathcal{M}}\left\|x_{m}\right\|_{\infty} .
$$

Согласно следствию 9.1 получаем $\lim _{t \rightarrow \pm \infty}\left\|\chi_{[t, \pm \infty)} w_{l m}\right\|_{\mathcal{M}}=0$. 
7.2. Доказательство утверждения 1) теоремы 2.2. Далее понадобятся следующие утверждения.

УтвЕРЖДЕНИЕ 7.1. Функиия $\mathcal{E}(t)=\mathcal{E}(\xi(\nu, t), \dot{\xi}(\nu, t))$, определенная в предложении 6.3, имеет вид

$$
\mathcal{E}(t)=\left\|\left\langle\widehat{a}, \widehat{x}^{t}\right\rangle+\sqrt{\rho} \xi_{\bullet}\right\|_{L_{2}}^{2} .
$$

ДокАЗАтЕЛЬСтво. Прямым вычислением из (7.3) получаем $\mathcal{E}=\mathcal{E}_{22}+\mathcal{E}_{21}+$ $\mathcal{E}_{11}$, где

$$
\begin{gathered}
\mathcal{E}_{22}(t):=\int \rho\left(\nu^{2} \xi_{2}^{2}+\dot{\xi}_{2}^{2}\right) d \nu=\left\|\sqrt{\rho} \xi_{\bullet}\right\|_{L_{2}}^{2}, \\
\mathcal{E}_{21}(t):=\int \rho\left(\nu^{2} \xi_{2} \xi_{1}+\dot{\xi}_{2} \dot{\xi}_{1}\right) d \nu=\int\left\langle\widehat{a}, \widehat{x}^{t}\right\rangle \overline{\sqrt{\rho} \xi_{\bullet}} d \nu+\int \overline{\left\langle\widehat{a}, \widehat{x}^{t}\right\rangle} \sqrt{\rho} \xi_{\bullet} d \nu, \\
\mathcal{E}_{11}(t):=\int \rho\left(\nu^{2} \xi_{1}^{2}+\dot{\xi}_{1}^{2}\right) d \nu=\left\|\left\langle\widehat{a}, \widehat{x}^{t}\right\rangle\right\|_{L_{2}}^{2} .
\end{gathered}
$$

Утверждение доказано.

СледСТвиЕ 7.2. Имеем $\left\|\left\langle\widehat{a}, \widehat{x}^{t}\right\rangle\right\|_{L_{2}}^{2}<c_{5}(E)$.

ДокАЗАТЕЛЬство. Из предложения 6.3 следует, что $\mathcal{E}(t) \leqslant c_{4}(E)$. Согласно утверждению 7.1 получаем

$$
\mathcal{E}(t)=\int\left|\sqrt{\rho} \xi_{\bullet}+\left\langle\widehat{a}, \widehat{x}^{t}\right\rangle\right|^{2} d \nu<c_{4}(E) .
$$

Из Н4 вытекает, что $\sqrt{\rho} \xi_{\bullet} \in L_{2}$. Следствие доказано.

В следствии 7.2 заключена основная идея доказательства: если $\left\langle\widehat{a}, \widehat{x}^{t}\right\rangle \in L_{2}$ при всех $t$, то $\widehat{x}$ не может содержать сингулярностей на частотах $\nu \notin\left(-\nu_{0}, \nu_{0}\right)$ (см. предположение Н6, 1)). Далее мы это показываем строго.

1. Семейство обобщенных функций $\widehat{a}_{l} \widehat{x}_{l}^{t} \in \widehat{\mathcal{C}}_{b}$, рассматриваемых как функционалы на $\widehat{\mathcal{M}}$, при $t \rightarrow+\infty$ стремится к

$$
\widehat{a}_{l} \widehat{x}_{l}^{+}:=\widehat{a}_{l} \mathcal{F}\left(\chi_{[0,+\infty]} x_{l}\right) \in \widehat{\mathcal{C}}_{b} .
$$

Действительно, из Н5 следует, что $a_{l}=\mathcal{F}^{-1}\left(\widehat{a}_{l}\right) \in \mathcal{M}$. Поэтому для любой функции $\widehat{\varphi}=\mathcal{F}(\varphi) \in \widehat{\mathcal{M}}$ выполнено $a_{l} * \bar{\varphi} \in \mathcal{M}$. Следовательно,

$$
\left(\widehat{a}_{l} \widehat{x}_{l}^{t}, \widehat{\varphi}\right)=2 \pi\left(\mathcal{F}^{-1}\left(\widehat{a}_{l} \widehat{x}_{l}^{t}\right), \varphi\right)=2 \pi \int\left(a_{l} * x_{l}^{t}\right) \bar{\varphi} d \tau=2 \pi \int x_{l}^{t}\left(a_{l} * \bar{\varphi}\right) d \tau .
$$

Из следствия 9.1 получаем, что при $t \rightarrow+\infty$ это выражение стремится к

$$
2 \pi \int x_{l}^{+}\left(a_{l} * \bar{\varphi}\right) d \tau=\left(\widehat{a}_{l} \widehat{x}_{l}^{+}, \widehat{\varphi}\right) .
$$

Отсюда следует, что $\left\langle\widehat{a}, \widehat{x}^{t}\right\rangle$ стремится к $\left\langle\widehat{a}, \widehat{x}^{+}\right\rangle$, где $\widehat{x}^{t}=\left(\widehat{x}_{1}^{t}, \ldots, \widehat{x}_{n}^{t}\right)$, а $\widehat{x}^{+}=$ $\left(\widehat{x}_{1}^{+}, \ldots, \widehat{x}_{n}^{+}\right)$.

2. Следствие 7.2 показывает, что $L_{2}$-норма выражения $\left\langle\widehat{a}, \widehat{x}^{+}\right\rangle$, рассматриваемого как функционал на $\widehat{\mathcal{M}} \cap L_{2} \subset L_{2}$, не превосходит $\sqrt{2 c_{3}(E)}$. Поскольку 
$\widehat{\mathcal{M}} \cap L_{2}$ плотно в $L_{2}$, по теореме Хана-Банаха $\left\langle\widehat{a}, \widehat{x}^{+}\right\rangle$может быть продолжено до функционала на $L_{2}$ с той же нормой. Этот функционал мы будем обозначать так же: $\left\langle\widehat{a}, \widehat{x}^{+}\right\rangle$. По теореме Рисса функционал $\left\langle\widehat{a}, \widehat{x}^{+}\right\rangle$может быть отождествлен с некоторым элементом из $L_{2}$.

Аналогично получаем, что $\left\langle\widehat{a}, \widehat{x}^{-}\right\rangle \in L_{2}$, где $\widehat{x}_{l}^{-}=\mathcal{F}\left(\chi_{(-\infty, 0]} x_{l}\right)$. Поскольку $\widehat{x}_{l}=\widehat{x}_{l}^{+}+\widehat{x}_{l}^{-}$, получаем $\langle\widehat{a}, \widehat{x}\rangle \in L_{2}$.

3. Рассмотрим уравнение (1.4):

$$
\ddot{x}=-B x+\int \kappa(\nu) \xi(\nu) d \nu .
$$

Сделаем его преобразование Фурье и используем уже доказанное утверждение 2) теоремы 2.2. Получим

$$
\left(B-\lambda^{2} E-\widehat{W}_{\diamond}^{\star}(\lambda)\right) \widehat{x}(\lambda)=\widehat{\vartheta}^{\star}(\lambda) \quad \text { или } \quad \widehat{V}^{\star}(\lambda) \widehat{x}=\widehat{\vartheta}^{\star},
$$

где $\widehat{V}^{\star}(\lambda)$ определено в $(2.4), \widehat{\vartheta}^{\star} \in \mathcal{C}_{0}^{n \star}, \star \in\{+,-\}$.

4. Сначала покажем, что $\operatorname{sing} \operatorname{supp}(\widehat{x}) \subset\left[-\nu_{0}, \nu_{0}\right]$. Предположим противное, т. е. существует $\lambda_{0} \in \operatorname{sing} \operatorname{supp}(\widehat{x}), \lambda_{0} \notin\left[-\nu_{0}, \nu_{0}\right]$. Для определенности будем считать, что $\lambda_{0} \in \operatorname{sing} \operatorname{supp}^{+}(\widehat{x})$.

В этом случае согласно (7.8) имеем $\widehat{V}^{+}(\lambda) \widehat{x}=\widehat{\vartheta}^{+}$, где $\widehat{\vartheta}^{+} \in \widehat{\mathcal{C}}_{0}^{n+}$. Домножим последнее соотношение на произвольную $\widehat{\mu}_{1} \in \widehat{\mathcal{M}}, \widehat{\mu}_{1}\left(\lambda_{0}\right) \neq 0, \operatorname{supp}\left(\widehat{\mu}_{1}\right)-$ компакт. Получим следующую систему:

$$
\langle\widehat{a}, \widehat{x}\rangle \in L_{2}, \quad\left(\widehat{\mu}_{1} \widehat{V}^{+}\right)(\lambda) \widehat{x}=0\left(\bmod \widehat{\mathcal{C}}_{0}^{n+}\right),
$$

где равенство по mod $\widehat{\mathcal{C}}_{0}^{n+}$ означает равенство с точностью до элемента из $\widehat{\mathcal{C}}_{0}^{n+}$. Преобразуем эту систему к более удобному виду.

а) Согласно (2.4) и предложению 2.1 элементы матрицы $\left(\widehat{\mu}_{1} \widehat{V}^{+}\right)$лежат в $\widehat{\mathcal{M}}$. Поэтому мы можем применить лемму 9.7: так как $\lambda_{0} \in \operatorname{sing}_{\operatorname{supp}}(\widehat{x})$, то $\operatorname{det}\left(\widehat{\mu}_{1} \widehat{V}^{+}\right)\left(\lambda_{0}\right)=0$, что равносильно $\operatorname{det} \widehat{V}^{+}\left(\lambda_{0}\right)=0$.

Согласно H6, 3) существует индекс $l$ такой, что $\widehat{a}_{l}\left(\lambda_{0}\right) \neq 0$. Из замечания 2.2 следует, что $\frac{\widehat{a}_{l}}{\nu} \in L_{2}$. Домножим первое соотношение (7.9) на $\frac{2 \pi \widehat{a}_{l}}{i \nu} \in L_{2}$; в результате получим $\frac{2 \pi \widehat{a}_{l}}{i \nu}\langle\widehat{a}, \widehat{x}\rangle=\left\langle\widehat{w}_{l}, \widehat{x}\right\rangle \in L_{1} \subset \widehat{\mathcal{C}}_{0}$.

b) Выберем достаточно малый интервал $I$. Поскольку

$$
\lambda_{0} \in \operatorname{sing} \operatorname{supp}^{+}(\widehat{x}), \quad \text { то } \exists \widehat{\mu} \in \widehat{\mathcal{M}}, \operatorname{supp} \widehat{\mu} \subset I: \widehat{\mu} \widehat{x} \notin \widehat{\mathcal{C}}_{0}^{n+} .
$$

Домножим оба соотношения (7.9) на $\widehat{\mu}$. Получим следующую систему:

$$
\left\langle\widehat{w}_{l}, \widehat{\mu} \widehat{x}\right\rangle=0 \quad\left(\bmod \widehat{\mathcal{C}}_{0}^{+}\right), \quad\left(\widehat{\mu}_{1} \widehat{V}^{+}\right)(\lambda)(\widehat{\mu} \widehat{x})=0 \quad\left(\bmod \widehat{\mathcal{C}}_{0}^{n+}\right), \quad \operatorname{det} \widehat{V}^{+}\left(\lambda_{0}\right)=0 .
$$

5. Теперь получим противоречие с условием Н6,3). Из Н6,2) следует, что $\lambda_{0}-$ изолированный нуль первого порядка уравнения $\operatorname{det} \widehat{V}^{+}\left(\lambda_{0}\right)=0$. Поэтому из леммы 9.8 следует, что

$$
\widehat{\mu} \widehat{x}=\widehat{\sigma} \widehat{y}\left(\bmod \widehat{\mathcal{C}}_{0}^{n+}\right),
$$

где $\widehat{\sigma} \in \widehat{\mathcal{M}}^{n}, \widehat{y} \in \widehat{\mathcal{C}_{b}}$, причем $\widehat{\sigma}\left(\lambda_{0}\right) \in \operatorname{Ker} \widehat{V}^{+}\left(\lambda_{0}\right)$. 
ПреДЛОЖЕниЕ 7.3. Если $\lambda_{0} \in \operatorname{sing} \operatorname{supp}^{+}(\widehat{x})$, mo $\lambda_{0} \in \operatorname{sing} \operatorname{supp}^{+}(\widehat{y})$.

Доказательство предложения 7.3 приведено после окончания доказательства теоремы.

Подставляя (7.12) в первое уравнение (7.11), получим

$$
\left\langle\widehat{w}_{l}, \widehat{\sigma}\right\rangle \widehat{y}=0 \quad\left(\bmod \widehat{\mathcal{C}}_{0}^{+}\right) .
$$

Применяя лемму 9.7 к одномерной матрице $\left\langle\widehat{w}_{l}, \widehat{\sigma}\right\rangle$, из (7.13) получаем, что

$$
\left\langle\widehat{w}_{l}\left(\lambda_{0}\right), \widehat{\sigma}\left(\lambda_{0}\right)\right\rangle=0 \quad \text { или } \quad \widehat{w}_{l}\left(\lambda_{0}\right) \perp \widehat{\sigma}\left(\lambda_{0}\right) .
$$

Поскольку $\widehat{\sigma}\left(\lambda_{0}\right) \in \operatorname{Ker} \widehat{V}^{+}\left(\lambda_{0}\right)$ и ядро матрицы $\widehat{V}^{+}\left(\lambda_{0}\right)$ одномерно, то

$$
\begin{aligned}
\widehat{w}_{l}\left(\lambda_{0}\right) \notin \operatorname{Ker} \widehat{V}^{+}\left(\lambda_{0}\right) & \Rightarrow \widehat{w}_{l}\left(\lambda_{0}\right) \in \operatorname{Im} \widehat{V}^{+}\left(\lambda_{0}\right) \\
& \Rightarrow \quad \widehat{a}\left(\lambda_{0}\right)=\frac{i \lambda_{0}}{2 \pi \widehat{a}_{l}\left(\lambda_{0}\right)} \widehat{w}_{l}\left(\lambda_{0}\right) \in \operatorname{Im} \widehat{V}^{+}\left(\lambda_{0}\right),
\end{aligned}
$$

так как мы выбрали $\widehat{a}_{l}\left(\lambda_{0}\right) \neq 0$. Это противоречит условию $\left.\mathrm{H} 6,3\right)$.

6. Осталось показать, что $\operatorname{sing} \operatorname{supp}(\widehat{x}) \cap\left\{ \pm \nu_{0}\right\}=\varnothing$. Согласно (7.8) и лемме 9.7 для того, чтобы $\left\{ \pm \nu_{0}\right\} \in \operatorname{sing} \operatorname{supp}(\widehat{x})$, необходимо $\operatorname{det} \widehat{V}^{\star}\left( \pm \nu_{0}\right)=0$ для какой-нибудь 夫 из $\{+,-\}$. Однако согласно Н6,4) это невозможно.

ДОКАЗАТЕЛЬСТВО ПРЕДЛОЖЕНИЯ 7.3. Рассмотрим произвольный интервал $J \supset \lambda_{0}$ и произвольный элемент $\widehat{\mu}_{2} \in \widehat{\mathcal{M}}, \operatorname{supp} \widehat{\mu}_{2} \subset J, \widehat{\mu}_{2}\left(\lambda_{0}\right) \neq 0$. Покажем, что $\widehat{\mu}_{2} \widehat{y} \notin \widehat{\mathcal{C}}_{0}^{+}$.

Выберем $\widehat{\mu}_{3} \in \widehat{\mathcal{M}},\left.\widehat{\mu}_{3}\right|_{\operatorname{supp} \widehat{\mu}}=1$, supp $\widehat{\mu}_{3}$ достаточно мал. Домножим обе части (7.12) на $\widehat{\mu}_{3}$. Получим $\widehat{\mu} \widehat{x}=\widehat{\mu}_{3} \widehat{\sigma} \widehat{y} \notin \mathcal{C}_{0}^{n+}$. Это значит, что $\widehat{\sigma}$ имеет компоненту $j$ такую, что $\widehat{\mu}_{3} \widehat{\sigma}_{j} \widehat{y} \notin \mathcal{C}_{0}^{+}$. Согласно лемме 9.4 имеем $\widehat{\mu}_{3} \widehat{\sigma}_{j}=\widehat{\mu}_{4} \widehat{\mu}_{2}$ для некоторой $\widehat{\mu}_{4} \in \widehat{\mathcal{M}}$. Поэтому $\widehat{\mu}_{4} \widehat{\mu}_{2} \widehat{y} \notin \mathcal{C}_{0}^{+}$, а значит, $\widehat{\mu}_{2} \widehat{y} \notin \mathcal{C}_{0}^{+}$.

\section{§ 8. Динамика термостата}

В настоящем параграфе мы исследуем финальную динамику термостата. Мы показываем, что термостат слабо выходит на такой же предельный режим, как и конечномерная подсистема.

ТеОРема 8.1. Пусть выполнены предположения $\mathrm{H} 1-\mathrm{H} 6 \quad u \quad \alpha_{l}, \lambda_{l} u k m a-$ кие же, как в теореме 3.1. Тогда для любой действительной функиии $\widehat{\varphi}(\nu)$ такой, что $\frac{\widehat{\varphi}}{\sqrt{\rho} \nu} \in L_{2} \cap \widehat{\mathcal{M}}$, существуют постоянные $\beta_{1}, \ldots, \beta_{k}$, при которых выполнено

$$
\int \xi \widehat{\varphi} d \nu=\sum_{l=1}^{k}\left(\beta_{l} e^{i \lambda_{l} t}+\bar{\beta}_{l} e^{-i \lambda_{l} t}\right)+\vartheta(t),
$$

где $\vartheta \in \mathcal{C}_{0}$. Коэфбициенты $\beta_{l}$ вычисляются по формуле

$$
\beta_{l}=\int \frac{\widehat{\varphi}\left\langle\alpha_{l}, \widehat{a}\right\rangle}{\sqrt{\rho}\left(\lambda^{2}-\lambda_{l}^{2}\right)} d \lambda .
$$


Таким образом, при $t \rightarrow \pm \infty$ динамика термостата слабо стремится к конечной линейной комбинации гармонических колебаний с частотами $\lambda_{l}$.

ДоказАтельСтво теоремы 8.1. Обозначим $\widehat{\mu}=\frac{2 \pi \widehat{\varphi} \widehat{a}}{i \sqrt{\rho} \nu} \in \widehat{\mathcal{M}}^{n}$, так как согласно Н5 имеем $\widehat{a} \in \widehat{\mathcal{M}}^{n}$.

В п. 7.1 была выведена формула из утверждения 2) теоремы 2.2:

$$
\int \kappa \xi d \nu=\mathcal{F}^{-1}\left(\widehat{W}_{\diamond}^{ \pm} \widehat{x}\right)+\vartheta^{ \pm}, \quad \vartheta^{ \pm} \in \mathcal{C}_{0}^{n \pm}
$$

Заменив $\kappa$ на $\widehat{\varphi}$, аналогично можно получить формулу ${ }^{8}$

$$
\int \widehat{\varphi} \xi d \nu=\mathcal{F}^{-1}\left\langle\widehat{\mu}_{\diamond}^{ \pm}, \widehat{x}\right\rangle+\vartheta_{1}^{ \pm}, \quad \vartheta_{1}^{ \pm} \in \mathcal{C}_{0}^{ \pm}, \quad \widehat{\mu}_{\diamond}^{ \pm}=\mathcal{F}\left(\mu_{\diamond}^{ \pm}\right),
$$

причем компоненты вектора $\mu_{\diamond}^{ \pm}$вычисляются следующим образом:

$$
\mu_{\diamond l}^{ \pm}(\tau)= \begin{cases}\frac{ \pm\left(\mu_{l}(\tau)-\mu_{l}(-\tau)\right)}{2}, & \text { если } \pm \tau>0, \\ 0, & \text { если } \pm \tau<0 .\end{cases}
$$

Поэтому (аналогично п. 7.1)

$$
\widehat{\mu}_{\diamond l}^{ \pm}(\nu)= \pm \frac{1}{4}\left(\widehat{\mu}_{l}(\nu)-\widehat{\mu}_{l}(-\nu)\right)+\frac{1}{2 \pi i} \text { v.p. } \int \frac{\lambda \widehat{\mu}_{l}(\lambda)}{\nu^{2}-\lambda^{2}} d \lambda .
$$

Из (8.5) вытекает, что $\mu_{\diamond}^{ \pm} \in \mathcal{M}^{n}$, следовательно, $\widehat{\mu}_{\diamond}^{ \pm} \in \widehat{\mathcal{M}}^{n}$.

Из теоремы 3.1 следует, что

$$
\widehat{x}=2 \pi \sum_{l=1}^{k}\left(\alpha_{l} \delta\left(\lambda-\lambda_{l}\right)+\bar{\alpha}_{l} \delta\left(\lambda+\lambda_{l}\right)\right)+\widehat{\vartheta}_{2}, \quad \widehat{\vartheta}_{2} \in \widehat{\mathcal{C}}_{0}^{n} .
$$

Подставляя это выражение в (8.4), получаем

$$
\begin{aligned}
\int \widehat{\varphi} \xi d \nu & =2 \pi \sum_{l=1}^{k} \mathcal{F}^{-1}\left(\left\langle\widehat{\mu}_{\diamond}^{ \pm}, \alpha_{l}\right\rangle \delta\left(\lambda-\lambda_{l}\right)+\left\langle\widehat{\mu}_{\diamond}^{ \pm}, \bar{\alpha}_{l}\right\rangle \delta\left(\lambda+\lambda_{l}\right)+\left\langle\widehat{\mu}_{\diamond}^{ \pm}, \widehat{\vartheta}_{2}\right\rangle\right)+\vartheta_{1}^{ \pm} \\
& =\sum_{l=1}^{k}\left(\left\langle\widehat{\mu}_{\diamond}^{ \pm}\left(\lambda_{l}\right), \alpha_{l}\right\rangle e^{i \lambda_{l} t}+\left\langle\widehat{\mu}_{\diamond}^{ \pm}\left(-\lambda_{l}\right), \bar{\alpha}_{l}\right\rangle e^{-i \lambda_{l} t}\right)+\vartheta^{ \pm}
\end{aligned}
$$

где $\vartheta^{ \pm}=\sum_{l=1}^{k} \mathcal{F}^{-1}\left\langle\widehat{\mu}_{\diamond}^{ \pm}, \widehat{\vartheta}_{2}\right\rangle+\vartheta_{1}^{ \pm} \in \mathcal{C}_{0}^{ \pm}$согласно $2,(\mathrm{~d})$ из п. 9.1.

Далее, из формулы (8.6) следует, что $\widehat{\mu}_{\diamond}^{+}\left(\lambda_{l}\right)=\widehat{\mu}_{\diamond}^{+}\left(-\lambda_{l}\right)=\widehat{\mu}_{\diamond}^{-}\left(\lambda_{l}\right)=\widehat{\mu}_{\diamond}^{-}\left(-\lambda_{l}\right)$ для всех $1 \leqslant l \leqslant k$, так как согласно H6, 1) имеем $\widehat{\mu}=\left.\frac{2 \pi \widehat{\varphi} \widehat{a}}{i \sqrt{\rho} \nu}\right|_{\left(-\nu_{0}, \nu_{0}\right)}=0, \pm \lambda_{l} \in$ $\left(-\nu_{0}, \nu_{0}\right)$. Поэтому финальная динамика системы на $+\infty$ и $-\infty$ одинакова.

С учетом последнего мы получаем формулу (8.2) прямой подстановкой соотношения (8.6) в (8.7).

\footnotetext{
${ }^{8}$ То обстоятельство, что в формуле (8.4) стоит скалярное произведение, а в формуле (8.3) умножение на матрицу, объясняется тем, что $\kappa$ - векторнозначная функция, а $\widehat{\varphi}-$ скалярнозначная.
} 


\section{§ 9. Пространства $\mathcal{C}_{b}, \mathcal{C}_{0}^{ \pm}, \mathcal{M}$ и их преобразования Фурье}

9.1. Определения. Здесь мы рассматриваем пространства $\mathcal{C}_{b}, \mathcal{C}_{0}^{ \pm}, \mathcal{M}$ и их преобразования Фурье и обсуждаем их основные свойства.

1. (а) Пусть $\mathcal{C}_{b}$ - пространство равномерно непрерывных ограниченных функций $\mathbb{R} \rightarrow \mathbb{C}$. Это банахово пространство с нормой из $L_{\infty}$ :

$$
\|\varphi\|_{\infty}=\sup |\varphi|, \quad \varphi \in \mathcal{C}_{b} .
$$

Кроме того, $\mathcal{C}_{b}$ - ассоциативная коммутативная банахова алгебра: для любых $\varphi, \psi \in \mathcal{C}_{b}$ выполнено $\varphi \psi \in \mathcal{C}_{b}$ и $\|\varphi \psi\|_{\infty} \leqslant\|\varphi\|_{\infty}\|\psi\|_{\infty}$.

(b) Пусть $\mathcal{C}_{0}^{+} \subset \mathcal{C}_{b}-$ пространство функций, стремящихся к нулю на $+\infty$, и $\mathcal{C}_{0}^{-} \subset \mathcal{C}_{b}-$ пространство функций, стремящихся к нулю на $-\infty$. Тогда $\mathcal{C}_{0}^{ \pm}-$ замкнутые подпространства и, кроме того, идеалы в $\left(\mathcal{C}_{b}, \cdot\right)$, т. е. $\mathcal{C}_{0}^{ \pm} \cdot \mathcal{C}_{b} \subset \mathcal{C}_{0}^{ \pm}$.

Пусть $\mathcal{C}_{0}=\mathcal{C}_{0}^{+} \cap \mathcal{C}_{0}^{-}$. Это также замкнутый идеал в $\mathcal{C}_{b}$.

(c) Пусть $\mathcal{M}$ - пространство плотностей конечных счетно-аддитивных радоновых $\mathbb{C}$-мер на $\mathbb{R}$. Эти плотности являются обобщенными функциями на $\mathbb{R}$. Для любого элемента $\mu \in \mathcal{M}$ соответствующая мера есть $\mu(\tau) d \tau$. Пространство $\mathcal{M} \subset \mathcal{C}_{b}^{*}$ является банаховым пространством с нормой

$$
\|\mu\|_{\mathcal{M}}=\sup _{\|\varphi\|_{\infty}=1} \mu(\varphi), \quad \mu(\varphi)=\int \varphi \mu d \tau, \quad \varphi \in \mathcal{C}_{b}, \quad \mu \in \mathcal{M} .
$$

Ясно, что $L_{1} \subset \mathcal{M}$.

Напомним, что $\mathcal{M}$ - сверточная банахова алгебра:

$$
\left\|\mu_{1} * \mu_{2}\right\|_{\mathcal{M}} \leqslant\left\|\mu_{1}\right\|_{\mathcal{M}}\left\|\mu_{2}\right\|_{\mathcal{M}} .
$$

Для любой $\mu \in \mathcal{M}$ существует разложение

$$
\mu=\mu_{1}-\mu_{2}+i \mu_{3}-i \mu_{4},
$$

где $\mu_{1}, \mu_{2}, \mu_{3}, \mu_{4} \in \mathcal{M}$ неотрицательны (см., например, [9]).

(d) Определена свертка элементов $\mu \in \mathcal{M}$ и $\varphi \in \mathcal{C}_{b}$. Рассмотрим линейный изометрический оператор

$$
\mathbf{s}_{t}: \mathcal{C}_{b} \rightarrow \mathcal{C}_{b}, \quad\left(\mathbf{s}_{t} \varphi\right)(\tau)=\varphi(t-\tau) .
$$

Тогда $(\mu * \varphi)(t)=\mu\left(\mathbf{s}_{t} \varphi\right)$. Согласно лемме 9.2 получим $\mathcal{M} * \mathcal{C}_{b} \subset \mathcal{C}_{b}, \mathcal{M} * \mathcal{C}_{0} \subset \mathcal{C}_{0}$ и

$$
\|\mu * \varphi\|_{\infty} \leqslant\|\mu\|_{\mathcal{M}}\|\varphi\|_{\infty} .
$$

2. (а) Положим $\widehat{\mathcal{C}}_{b}=\mathcal{F}\left(\mathcal{C}_{b}\right)$. Элементы пространства $\widehat{\mathcal{C}_{b}}$ являются обобщенными функциями. В частности, $\widehat{\mathcal{C}_{b}}$ содержит $\delta$-функцию. Соотношение $\mathcal{F}^{-1}(\widehat{\varphi} * \widehat{\psi})=2 \pi \varphi \psi$ показывает, что $\left(\widehat{\mathcal{C}}_{b}, *\right)$ - ассоциативная коммутативная алгебра: $\widehat{\varphi} * \widehat{\psi} \in \widehat{\mathcal{C}_{b}}$ для любых $\widehat{\varphi}, \widehat{\psi} \in \widehat{\mathcal{C}_{b}}$.

(b) Кроме того, $\widehat{\mathcal{C}}_{0}^{+}=\mathcal{F}\left(\mathcal{C}_{0}^{+}\right), \widehat{\mathcal{C}}_{0}^{-}=\mathcal{F}\left(\mathcal{C}_{0}^{-}\right)$и $\widehat{\mathcal{C}}_{0}=\mathcal{F}\left(\mathcal{C}_{0}\right)$ являются идеалами B $\left(\widehat{\mathcal{C}}_{b}, *\right)$.

(с) Положим $\widehat{\mathcal{M}}=\mathcal{F}(\mathcal{M})$. Нетрудно показать, что $\widehat{\mathcal{M}} \subset \mathcal{C}_{b}$, однако $\widehat{\mathcal{M}} \neq \mathcal{C}_{b}$ (см. [9]). Согласно теореме Римана-Лебега образ $\mathcal{F}\left(L_{1}\right)$ лежит в $\mathcal{C}_{0}$.

Согласно $1,\left(\right.$ с) имеем $\widehat{\mu}_{1} \widehat{\mu}_{2} \in \widehat{\mathcal{M}}$ для любых $\widehat{\mu}_{1}, \widehat{\mu}_{2} \in \widehat{\mathcal{M}}$.

(d) Согласно 1, (d) имеем $\widehat{\mathcal{M}} \cdot \widehat{\mathcal{C}_{b}} \subset \widehat{\mathcal{C}_{b}}, \widehat{\mathcal{M}} \cdot \widehat{\mathcal{C}}_{0}^{+} \subset \widehat{\mathcal{C}}_{0}^{+}, \widehat{\mathcal{M}} \cdot \widehat{\mathcal{C}}_{0}^{-} \subset \widehat{\mathcal{C}}_{0}^{-}$и $\widehat{\mathcal{M}} \cdot \widehat{\mathcal{C}_{0}} \subset \widehat{\mathcal{C}_{0}}$. 
3. (а) Пусть $\widehat{\varphi} \in \widehat{\mathcal{C}_{b}}, \lambda \in \mathbb{R}$. Будем говорить, что $\lambda \notin \operatorname{sing} \operatorname{supp}^{+}(\widehat{\varphi})$ в том и только в том случае, если существует интервал $I \ni \lambda$ такой, что для любого $\widehat{\mu} \in \widehat{\mathcal{M}}, \operatorname{supp} \widehat{\mu} \subset I$, имеет место включение $\widehat{\mu} \widehat{\varphi} \in \widehat{\mathcal{C}}_{0}^{+}$.

(b) Обратно, для $\widehat{\varphi} \in \widehat{\mathcal{C}_{b}}$ будем говорить, что $\lambda \in \operatorname{sing} \operatorname{supp}^{+}(\widehat{\varphi})$ в том и только в том случае, если для любого интервала $I \ni \lambda$ существует $\widehat{\mu} \in \widehat{\mathcal{M}}$ такое, что $\operatorname{supp} \widehat{\mu} \subset I$ и $\widehat{\mu} \widehat{\varphi} \in \widehat{\mathcal{C}}_{b} \backslash \widehat{\mathcal{C}}_{0}^{+}$.

Аналогично определим sing $\operatorname{supp}^{-}(\widehat{\varphi})$. Ясно, что множества $\operatorname{sing} \operatorname{supp}^{+}(\widehat{\varphi})$,

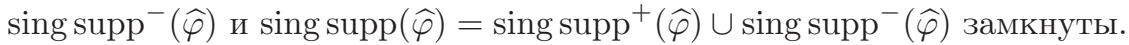

4. (а) Будем использовать следующие обозначения: $\mathcal{C}_{b}^{n}-$ пространство, состоящее из элементов вида $\left(\varphi_{1}, \ldots, \varphi_{n}\right)^{T}$, где $\varphi_{l} \in \mathcal{C}_{b}$, а $T$ обозначает транспонирование.

Аналогично определяются пространства $\mathcal{C}_{0}^{n}, \mathcal{C}_{0}^{n \pm}, \mathcal{M}^{n}$, а также их преобразования Фурье.

(b) Будем использовать следующее: например, для $\widehat{\mu} \in \widehat{\mathcal{M}}$ и $\widehat{x} \in \widehat{\mathcal{C}}_{b}^{n}$ запись $\widehat{\mu} \widehat{x}$ означает $\left(\widehat{\mu} \widehat{x}_{1}, \ldots, \widehat{\mu} \widehat{x}_{n}\right)^{T}$.

9.2. Леммы о $\mathcal{C}_{b}, \mathcal{C}_{0}^{ \pm}, \mathcal{M}$ и их преобразованиях Фурье. Сначала введем некоторые обозначения. Рассмотрим функции

$$
h_{0}(\tau)=\left\{\begin{array}{ll}
1-|\tau|, & \text { если }|\tau| \leqslant 1, \\
0, & \text { если }|\tau| \geqslant 1,
\end{array} \quad h_{k}(\tau)=h_{0}(k-\tau), \quad k \in \mathbb{Z} .\right.
$$

Очевидно, $h_{k} \in \mathcal{C}_{0}$ и $\sum_{k \in \mathbb{Z}} h_{k}=1$.

Для любого $K \in \mathbb{N}$ положим

$$
\mathbf{h}_{K}^{<}=\sum_{|k| \leqslant K} h_{k}, \quad \mathbf{h}_{K}^{>}=\sum_{|k|>K} h_{k} .
$$

Лемма 9.1. Пусть $\mu \in \mathcal{M}$. Тогда для любого $\varepsilon>0$ существует $N>0$ такое, что $|\mu(\varphi)|<\varepsilon\|\varphi\|_{\infty}$ для любого $\varphi \in \mathcal{C}_{b}$, удовлетворяющего условию $\operatorname{supp} \varphi \cap[-N, N]=\varnothing$.

ДокАЗАтЕльство. Согласно представлению (9.1) достаточно рассматривать неотрицательные $\mu$. Тогда

$$
\|\mu\|_{\mathcal{M}}=\mu(1)=\sum_{k \in \mathbb{Z}} \mu\left(h_{k}\right)
$$

Последний ряд сходится. Поэтому для любого $\varepsilon>0$ существует $K \in \mathbb{N}$ такое, что $\mu\left(\mathbf{h}_{K}^{>}\right)<\varepsilon$. Для любого $\varphi \in \mathcal{C}_{b}$ такого, что $\operatorname{supp} \varphi \cap[-K-2, K+2]=\varnothing$, получаем

$$
|\mu(\varphi)|=\left|\mu\left(\mathbf{h}_{K}^{>} \varphi\right)\right| \leqslant\|\varphi\|_{\infty}\left|\mu\left(\mathbf{h}_{K}^{>}\right)\right|<\varepsilon\|\varphi\|_{\infty} .
$$

Лемма доказана.

СлЕДСТВиЕ 9.1. Для любого $\mu \in \mathcal{M}$ выполнено

$$
\lim _{t \rightarrow+\infty}\left\|\chi_{[t,+\infty)} \mu\right\|_{\mathcal{M}}=\lim _{t \rightarrow-\infty}\left\|\chi_{(-\infty, t]} \mu\right\|_{\mathcal{M}}=0 .
$$


ДокАЗАТЕЛьство. Поскольку $\mu$ можно считать неотрицательным, имеем

$$
\left\|\chi_{[t,+\infty)} \mu\right\|_{\mathcal{M}}=\left(\chi_{[t,+\infty)} \mu\right)(1) \leqslant \mu(\tilde{h}), \quad \tilde{h}=\sum_{k=[t]-1}^{+\infty} h_{k} .
$$

Остается заметить, что $\operatorname{supp}(\tilde{h}) \cap(-t+2, t-2)=\varnothing,\|\tilde{h}\|_{\infty}=1$. Поэтому согласно лемме 9.1 имеем $\lim _{t \rightarrow+\infty}\left\|\chi_{[t,+\infty)} \mu\right\|_{\mathcal{M}}=0$. Второй предел вычисляется аналогично.

Лемма 9.2. Для любъх $\varphi \in \mathcal{C}_{b} u \mu \in \mathcal{M}$ имеем $\mu * \varphi \in \mathcal{C}_{b} u\|\mu * \varphi\|_{\infty} \leqslant$ $\|\mu\|_{\mathcal{M}}\|\varphi\|_{\infty}$. Кроме того, если $\varphi \in \mathcal{C}_{0}^{\star}, \star \in\{+,-\}$, mо $\mu * \varphi \in \mathcal{C}_{0}^{\star}$.

ДокАзАТЕЛЬСтво. Функция $\mu * \varphi$ ограничена, так как

$$
|(\mu * \varphi)(t)|=\left|\mu\left(\mathbf{s}_{t} \varphi\right)\right| \leqslant\|\mu\|_{\mathcal{M}}\|\varphi\|_{\infty} .
$$

Поскольку $\varphi$ равномерно непрерывна, для любого $\varepsilon>0$ существует $\alpha>0$ такое, что $\left|\mathbf{s}_{t+\alpha} \varphi-\mathbf{s}_{t} \varphi\right|<\varepsilon$ для любого $t$. Тогда

$$
|(\mu * \varphi)(t+\alpha)-(\mu * \varphi)(t)|=\left|\mu\left(\mathbf{s}_{t+\alpha} \varphi-\mathbf{s}_{t} \varphi\right)\right| \leqslant\|\mu\|_{\mathcal{M} \varepsilon}
$$

Отсюда следует равномерная непрерывность $\varphi * h$.

Теперь предположим, что $\varphi \in \mathcal{C}_{0}^{+}$. Выберем произвольное $\varepsilon>0$. Согласно лемме 9.1 существует $N>1$ такое, что

$$
|\mu(\psi)|<\varepsilon\|\psi\|_{\infty} \quad \text { для } \quad \psi \in \mathcal{C}_{b}: \operatorname{supp} \psi \cap(-N-1, N+1)=\varnothing .
$$

Если понадобится, мы можем выбрать большее $N$ такое, что

$$
|\varphi(\tau)|<\varepsilon \quad \forall \tau>N
$$

Получаем $(\mu * \varphi)(t)=\mu\left(\mathbf{s}_{t} \varphi\right)=\mu\left(\mathbf{h}_{N}^{<} \mathbf{s}_{t} \varphi\right)+\mu\left(\mathbf{h}_{N}^{>} \mathbf{s}_{t} \varphi\right)$. Оценим последние два члена отдельно.

Для $t>2 N$ согласно (9.3) имеем $\left\|\mathbf{h}_{N}^{<} \mathbf{s}_{t} \varphi\right\|_{\infty}<\varepsilon$, поэтому

$$
\left|\mu\left(\mathbf{h}_{N}^{<} \mathbf{s}_{t} \varphi\right)\right|<\varepsilon\|\mu\|_{\mathcal{M}} .
$$

$\mathrm{C}$ другой стороны, $\operatorname{supp}\left(\mathbf{h}_{N}^{>} \mathbf{s}_{t} \varphi\right) \cap(-N-1, N+1)=\varnothing$. Следовательно,

$$
\left|\mu\left(\mathbf{h}_{N}^{>} \mathbf{s}_{t} \varphi\right)\right|<\varepsilon\left\|\mathbf{h}_{N}^{>} \mathbf{s}_{t} \varphi\right\|_{\infty} \leqslant \varepsilon\|\varphi\|_{\infty} .
$$

Комбинируя оценки (9.4) и (9.5), получим $|(\mu * \varphi)(t)| \leqslant \varepsilon\left(\|\mu\|_{\mathcal{M}}+\|\varphi\|_{\infty}\right)$.

Случай $\varphi \in \mathcal{C}_{0}^{-}$рассматривается аналогично.

СледСтвиЕ 9.2. Для любых $\widehat{\varphi} \in \widehat{\mathcal{C}_{b}}, \widehat{\varphi}_{0} \in \widehat{\mathcal{C}}_{0}^{\star}, \star \in\{+,-\}, u \widehat{\mu} \in \widehat{\mathcal{M}}$ выполнено $\widehat{\mu} \widehat{\varphi} \in \widehat{\mathcal{C}}_{b} u \widehat{\mu} \widehat{\varphi}_{0} \in \widehat{\mathcal{C}}_{0}^{\star}$.

Лемма 9.3. Пусть $\widehat{\beta} \in \widehat{\mathcal{M}}, \widehat{\beta}(0)=1$ u $\widehat{\beta}_{r}(\lambda)=\widehat{\beta}(r \lambda)$. Тогда для любого $\widehat{\varphi} \in \widehat{\mathcal{C}}_{b}$ выполнено

$$
\lim _{r \rightarrow 0}\left\|\mathcal{F}^{-1}\left(\widehat{\beta}_{r} \widehat{\varphi}\right)-\varphi\right\|_{\infty}=0 .
$$


ДоказАтЕльство. Сначала заметим, что $\beta_{r}(\varphi)=\beta\left(\varphi_{r}\right)$ для любой $\varphi \in \mathcal{C}_{b}$, где $\varphi_{r}(\tau)=\varphi(r \tau)$. Выберем произвольное $\varepsilon>0$. Существует $b>0$ такое, что

$$
|\varphi(t-\tau)-\varphi(t)|<\varepsilon \quad \forall t \in \mathbb{R}, \quad|\tau| \leqslant b .
$$

Согласно лемме 9.1 можно выбрать $r_{0}>0$ такое, что

$$
|\beta(\varphi)|<\varepsilon\|\varphi\|_{\infty} \quad \forall \varphi \in \mathcal{C}_{b}: \quad \operatorname{supp}(\varphi) \cap\left[2-\frac{b}{r_{0}},-2+\frac{b}{r_{0}}\right]=\varnothing .
$$

Далее мы предполагаем, что $0<r<r_{0}$. Выберем целое $K \in[-2+b / r,-1+b / r]$. Получим

$$
\begin{aligned}
\left(\beta_{r} * \varphi\right)(t) & =\beta_{r}\left(\mathbf{s}_{t} \varphi\right)=\beta\left(\mathbf{s}_{t / r} \varphi_{r}\right) \\
& =\varphi(t) \beta\left(\mathbf{h}_{K}^{<}\right)+\beta\left(\mathbf{h}_{K}^{<}\left(\mathbf{s}_{t / r} \varphi_{r}-\varphi(t)\right)\right)+\beta\left(\mathbf{h}_{K}^{>} \mathbf{s}_{t / r} \varphi_{r}\right) .
\end{aligned}
$$

Имеют место следующие оценки:

1) поскольку $\beta(1)=1$ и

$$
\operatorname{supp}\left(\mathbf{h}_{K}^{>}\right) \cap\left[-2-\frac{b}{r}, 2+\frac{b}{r}\right]=\varnothing,
$$

неравенство (9.7) влечет

$$
\left|\beta\left(\mathbf{h}_{K}^{<}\right)-1\right|=\left|\beta\left(\mathbf{h}_{K}^{>}\right)\right| \leqslant \varepsilon\left\|\mathbf{h}_{K}^{>}\right\|_{\infty}=\varepsilon ;
$$

2) согласно (9.6) имеем

$$
\left|\left(\mathbf{s}_{t / r} \varphi_{r}\right)(\tau)-\varphi(t)\right|<\varepsilon \quad \forall|\tau| \leqslant \frac{b}{r}
$$

неравенство $K \leqslant b / r_{0}-1$ показывает, что $\left\|\mathbf{h}_{K}^{<}\left(\mathbf{s}_{t / r} \varphi_{r}-\varphi(t)\right)\right\|_{\infty}<\varepsilon$, таким образом, получаем

$$
\left|\beta\left(\mathbf{h}_{K}^{<}\left(\mathbf{s}_{t / r} \varphi_{r}-\varphi(t)\right)\right)\right| \leqslant\|\beta\|_{\mathcal{M}}\left\|\mathbf{h}_{K}^{<}\left(\mathbf{s}_{t / r} \varphi_{r}-\varphi(t)\right)\right\|_{\infty} \leqslant \varepsilon\|\beta\|_{\mathcal{M}}
$$

3) используя (9.8), получаем

$$
\left|\beta\left(\mathbf{h}_{K}^{>} \mathbf{s}_{t / r} \varphi_{r}\right)\right| \leqslant \varepsilon\|\varphi\|_{\infty} .
$$

Из комбинации оценок (9.9)-(9.11) следует

$$
\left|\left(\beta_{r} * \varphi\right)(t)-\varphi(t)\right|<\varepsilon\left(2\|\varphi\|_{\infty}+\|\beta\|_{\mathcal{M}}\right) .
$$

Лемма 9.4. Пусть $\widehat{\mu} \in \widehat{\mathcal{M}}, \widehat{\mu}(\lambda) \neq 0, u I$ - достаточно малый интервал, содержащий $\lambda$. Тогда:

(а) для любого $\widehat{\mu}_{0} \in \widehat{\mathcal{M}}$, supp $\widehat{\mu}_{0} \subset I$, выполнено $\widehat{\mu}_{0}=\widehat{\mu} \widehat{\mu}_{1}$ для некоторого $\widehat{\mu}_{1} \in \widehat{\mathcal{M}}$

(b) для любого $\widehat{\varphi} \in \widehat{\mathcal{C}}_{b}, \operatorname{supp} \widehat{\varphi} \subset I$, выполнено $\widehat{\varphi}=\widehat{\mu} \widehat{\varphi}_{1}$ для некоторой $\widehat{\varphi}_{1} \in \widehat{\mathcal{C}_{b}}$. 
ДокАЗАтельство. Без ограничения общности считаем, что $\lambda=0$. Предположим, что supp $\widehat{\mu}_{0}=[-\alpha, \alpha] \subset I$. Ниже $\alpha$ будет выбрана достаточно малой. Положим $\beta=\chi_{[-1,1]}$ и $\beta_{\varepsilon}(\tau)=\beta(\varepsilon \tau)$. Как обычно, $\widehat{\beta}_{\varepsilon}=\mathcal{F}\left(\beta_{\varepsilon}\right)$. Тогда

$$
\begin{aligned}
\frac{\widehat{\mu}_{0}}{\widehat{\mu}} & =\frac{\widehat{\mu}_{0}}{\widehat{\beta}_{\varepsilon} * \widehat{\mu}-\left(\widehat{\beta}_{\varepsilon} * \widehat{\mu}-\widehat{\mu}\right)} \\
& =\frac{\widehat{\mu}_{0}}{\widehat{\beta}_{\varepsilon} * \widehat{\mu}}+\frac{\widehat{\mu}_{0}}{\left(\widehat{\beta}_{\varepsilon} * \widehat{\mu}\right)^{2}}\left(\widehat{\beta}_{\varepsilon} * \widehat{\mu}-\widehat{\mu}\right)+\frac{\widehat{\mu}_{0}}{\left(\widehat{\beta}_{\varepsilon} * \widehat{\mu}\right)^{3}}\left(\widehat{\beta}_{\varepsilon} * \widehat{\mu}-\widehat{\mu}\right)^{2}+\cdots
\end{aligned}
$$

Пусть $\hat{h}-$ гладкая функция такая, что $\left.\hat{h}\right|_{[-1,1]}=1$ и $\operatorname{supp}(\hat{h}) \subset[-2,2]$. Тогда $\hat{h}_{\alpha}(\lambda)=\hat{h}\left(\frac{\lambda}{\alpha}\right), \quad \hat{f}_{\varepsilon, \alpha}=\frac{\hat{h}_{\alpha}}{\widehat{\beta}_{\varepsilon} * \widehat{\mu}}, \quad f_{\varepsilon, \alpha}=\mathcal{F}^{-1}\left(\hat{f}_{\varepsilon, \alpha}\right), \quad g_{\varepsilon}=\mathcal{F}^{-1}\left(\widehat{\mu}-\widehat{\beta}_{\varepsilon} * \widehat{\mu}\right)$.

Пусть $\left.\hat{h}\right|_{\operatorname{supp}\left(\widehat{\mu}_{0}\right)}=1$. Тогда

$$
\mathcal{F}^{-1}\left(\frac{\widehat{\mu}_{0}}{\widehat{\mu}}\right)=\sum_{k=0}^{\infty} \mathcal{F}^{-1}\left(\frac{\widehat{\mu}_{0}\left(\hat{h}_{\alpha}\right)^{k+1}}{\left(\widehat{\beta}_{\varepsilon} * \widehat{\mu}\right)^{k+1}}\right) * \mathcal{F}^{-1}\left(\left(\widehat{\mu}-\widehat{\beta}_{\varepsilon} * \widehat{\mu}\right)^{k}\right)=\sum_{k=0}^{\infty} \mu_{0} * f_{\varepsilon, \alpha}^{* k} * g_{\varepsilon}^{* k} .
$$

Если интервал $I$ и $\varepsilon>0$ достаточно малы, то $\left.\left(\widehat{\beta}_{\varepsilon} * \widehat{\mu}\right)\right|_{I}$ близко к $\widehat{\mu}(0) \neq 0$. Кроме того, так как функции $\widehat{\beta}_{\varepsilon}$ и $\hat{h}_{\alpha}$ гладкие, то $\hat{f}_{\varepsilon, \alpha}$ также гладкая и $f_{\varepsilon, \alpha}$ быстро убывает. Поскольку $\operatorname{supp}\left(\hat{h}_{\alpha}\right)$ компактен, функция $f_{\varepsilon, \alpha}$ гладкая. Отсюда вытекают следующие соотношения:

$$
\lim _{\varepsilon \rightarrow+0}\left\|g_{\varepsilon}\right\|_{\mathcal{M}}=0, \quad \lim _{\alpha \rightarrow+0}\left\|f_{\varepsilon, \alpha}\right\|_{\mathcal{M}}=\left|b_{\varepsilon}\right|\|h\|_{\mathcal{M}} \quad \forall \varepsilon>0,
$$

где $1 / b_{\varepsilon}=\left(\widehat{\beta}_{\varepsilon} * \widehat{\mu}\right)(0)$. Мы докажем это несколькими строками ниже.

Согласно (9.12) можно выбрать $\varepsilon, \alpha>0$ такие, что $\left\|f_{\varepsilon, \alpha}\right\|_{\mathcal{M}}\left\|g_{\varepsilon}\right\|_{\mathcal{M}} \leqslant 1 / 2$. Оценка

$$
\left\|\mathcal{F}^{-1}\left(\widehat{\mu}_{0} / \widehat{\mu}\right)\right\|_{\mathcal{M}} \leqslant \sum_{k=0}^{\infty}\left\|\mu_{0}\right\|_{\mathcal{M}}\left\|f_{\varepsilon, \alpha}\right\|_{\mathcal{M}}^{k}\left\|g_{\varepsilon}\right\|_{\mathcal{M}}^{k}<\infty
$$

завершает доказательство п. (а).

Доказательство п. (b) аналогично, конечная оценка имеет следующий вид:

$$
\left\|\mathcal{F}^{-1}(\widehat{\varphi} / \widehat{\mu})\right\|_{\infty} \leqslant \sum_{k=0}^{\infty}\|\varphi\|_{\infty}\left\|f_{\varepsilon, \alpha}\right\|_{\mathcal{M}}^{k}\left\|g_{\varepsilon}\right\|_{\mathcal{M}}^{k}<\infty
$$

Теперь докажем (9.12). Первое соотношение вытекает из следствия 9.1. Докажем второе:

$$
\hat{f}_{\varepsilon, \alpha}=b_{\varepsilon} \hat{h}_{\alpha}+\hat{J} \hat{h}_{\alpha}, \quad \hat{J}=\frac{1}{\widehat{\beta}_{\varepsilon} * \widehat{\mu}}-b_{\varepsilon} .
$$

Следовательно,

$$
\left\|f_{\varepsilon, \alpha}\right\|_{\mathcal{M}} \leqslant\left\|b_{\varepsilon} h_{\alpha}\right\|_{\mathcal{M}}+\left\|\mathcal{F}^{-1}\left(\hat{J} \hat{h}_{\alpha}\right)\right\|_{\mathcal{M}}
$$

Функция $\hat{J}$ имеет компактный носитель и является гладкой в малой окрестности нуля. Поскольку нас интересует поведение $\hat{J}$ только в малой окрестности 
нуля, без ограничения общности будем считать, что $\hat{J}=\lambda \hat{J}_{1}$ для любого $\lambda \in \mathbb{R}$, где $\hat{J}_{1}$ гладкая и имеет компактный носитель. (Вообще говоря, нам достаточно, чтобы $\left\|J_{1}\right\|_{\mathcal{M}}<\infty$.) Поэтому

$$
\left\|\mathcal{F}^{-1}\left(\hat{J} \hat{h}_{\alpha}\right)\right\|_{\mathcal{M}}=\left\|J_{1} * \mathcal{F}^{-1}\left(\lambda \hat{h}_{\alpha}\right)\right\|_{\mathcal{M}} \leqslant\left\|J_{1}\right\|_{\mathcal{M}}\left\|\mathcal{F}^{-1}\left(\lambda \hat{h}_{\alpha}\right)\right\|_{\mathcal{M}}
$$

Остается заметить, что

$$
\mathcal{F}^{-1}\left(\lambda \hat{h}_{\alpha}\right)(\tau)=\alpha^{2} \mathcal{F}^{-1}(\lambda \hat{h})(\alpha \tau)
$$

Следовательно, $\left\|\mathcal{F}^{-1}\left(\lambda \hat{h}_{\alpha}\right)\right\|_{\mathcal{M}}=\alpha\left\|\mathcal{F}^{-1}(\lambda \hat{h})\right\|_{\mathcal{M}}$. С учетом $\left\|h_{\alpha}\right\|_{\mathcal{M}}=\|h\|_{\mathcal{M} \text { из }}$ соотношения (9.13) получаем

$$
\left\|f_{\varepsilon, \alpha}\right\|_{\mathcal{M}} \leqslant\left|b_{\varepsilon}\right|\|h\|_{\mathcal{M}}+\alpha\left\|J_{1}\right\|_{\mathcal{M}}\left\|\mathcal{F}^{-1}(\lambda \hat{h})\right\|_{\mathcal{M}}
$$

9.3. Леммы о сингулярном носителе. Рассмотрим детальнее свойства элементов из $\widehat{\mathcal{C}_{b}}$ в зависимости от их сингулярного носителя.

Лемма 9.5. Пусть $\widehat{\varphi} \in \widehat{\mathcal{C}_{b}}, \star \in\{+,-\} u \operatorname{sing} \operatorname{supp}^{\star}(\widehat{\varphi})=\varnothing$. Тогдa $\widehat{\varphi} \in \widehat{\mathcal{C}}_{0}^{\star}$.

ДоказАтельство. Пусть $\widehat{\beta} \in \widehat{\mathcal{M}}, \widehat{\beta}(0)=1, \operatorname{supp}(\widehat{\beta}) \subset[-1,1]$, и пусть $\widehat{\beta}_{r}(\lambda)=\widehat{\beta}(r \lambda)$. Рассмотрим произвольное $\varepsilon>0$. Тогда согласно лемме 9.3 для достаточно малого $r$ имеем

$$
\left\|\mathcal{F}^{-1}(\widehat{\beta} r \widehat{\mu} \widehat{\varphi})-\mathcal{F}^{-1}(\widehat{\mu} \widehat{\varphi})\right\|_{\infty}<\varepsilon
$$

Множество $\operatorname{supp}\left(\widehat{\beta}_{r} \widehat{\varphi}\right) \subset[-1 / r, 1 / r]$ компактно. Следовательно, $\widehat{\beta}_{r} \widehat{\varphi} \in \widehat{\mathcal{C}}_{0}^{\star}$. (Действительно, достаточно выбрать конечное покрытие отрезка $[-1 / r, 1 / r]$ интервалами $I$ из определения $3,\left(\right.$ а) в п. 9.1: $[-1 / r, 1 / r] \subset \bigcup_{j=1}^{N} I_{j}$, и воспользоваться равенством $\widehat{\beta}_{r} \widehat{\varphi}=\sum_{j=1}^{n} \widehat{\mu}_{j} \widehat{\beta}_{r} \widehat{\varphi}$, где $\left.\operatorname{supp} \widehat{\mu}_{j} \subset I_{j}.\right)$ Поэтому для достаточно большого $\star \tau>0$

$$
\left|\mathcal{F}^{-1}(\widehat{\mu} \widehat{\varphi})(\tau)\right| \leqslant\left\|\mathcal{F}^{-1}\left(\widehat{\beta}_{r} \widehat{\mu} \widehat{\varphi}\right)-\mathcal{F}^{-1}(\widehat{\mu} \widehat{\varphi})\right\|_{\infty}+\left|\left(\widehat{\beta}_{r} \widehat{\mu} \widehat{\varphi}\right)(\tau)\right| \leqslant \varepsilon .
$$

СледСтвиЕ 9.3. Пусть $\widehat{\varphi} \in \widehat{\mathcal{C}_{b}}, \widehat{\mu} \in \widehat{\mathcal{M}} u \operatorname{sing} \operatorname{supp}^{\star}(\widehat{\varphi}) \cap \operatorname{supp} \widehat{\mu}=\varnothing, \star \epsilon$ $\{+,-\}$. Тогда $\widehat{\mu} \widehat{\varphi} \in \widehat{\mathcal{C}}_{0}^{\star}$.

ДоказАтельство. Рассмотрим произвольное $\lambda \in \mathbb{R}$.

Пусть $\lambda \notin \operatorname{sing} \operatorname{supp}^{\star}(\widehat{\varphi})$. Тогда существует интервал $I$ такой, что для любого $\widehat{\nu} \in \widehat{\mathcal{M}}$ выполнено $\widehat{\nu} \widehat{\varphi} \in \widehat{\mathcal{C}}_{0}^{\star}$. Следовательно, $\widehat{\nu} \widehat{\mu} \widehat{\varphi} \in \widehat{\mathcal{C}}_{0}^{\star}$. Значит, $\lambda \notin \operatorname{sing} \operatorname{supp}^{\star}(\widehat{\mu} \widehat{\varphi})$.

Пусть $\lambda \in \operatorname{sing} \operatorname{supp}^{\star}(\widehat{\varphi})$. Тогда $\lambda \notin \operatorname{supp} \widehat{\mu}$. Значит, существует интервал $I \ni \lambda$ такой, что $I \cap \operatorname{supp} \widehat{\mu}=\varnothing$. Выберем произвольное $\widehat{\nu} \in \widehat{\mathcal{M}}$ такое, что $\operatorname{supp} \widehat{\nu} \in I$. Тогда $\widehat{\nu} \widehat{\mu}=0$ и $\widehat{\nu} \widehat{\mu} \widehat{\varphi}=0 \in \widehat{\mathcal{C}}_{0}^{\star}$. Следовательно, $\lambda \notin \operatorname{sing} \operatorname{supp}^{\star}(\widehat{\mu} \widehat{\varphi})$.

Таким образом, sing $\operatorname{supp}^{\star}(\widehat{\mu} \widehat{\varphi})=\varnothing$. Для завершения доказательства остается применить лемму 9.5 . 
9.4. Леммы о системах уравнений. Справедливы следующие утверждения.

Лемма 9.6. Пусть матрица $\widehat{M}$ размера $n \times n$ состоит из элементов $\widehat{\mu}_{i j} \in$ $\widehat{\mathcal{M}} u \operatorname{det} \widehat{M}\left(\lambda_{0}\right) \neq 0$ в некоторой точке $\lambda_{0}$. Тогда на некотором достаточно малом интервале $I, \lambda_{0} \in I$, определена матрица $\widehat{M}^{-1}$ с элементами из $\widehat{\mathcal{M}}$ такая, что $\widehat{M}^{-1} \widehat{M}=E$ в любой точке $\lambda \in I$.

ДокАЗАТЕЛЬство. В некоторой окрестности точки $\lambda_{0}$ имеем $\operatorname{det} \widehat{M} \neq 0$, так как элементы матрицы $\widehat{M}$ лежат в $\widehat{\mathcal{M}}$, а $\widehat{\mathcal{M}} \in \mathcal{C}_{b}$. Согласно стандартной формуле элементы обратной матрицы $\widehat{\sigma}_{i j}$ имеют вид $\widehat{\sigma}_{i j}=\frac{\widehat{\mu} \hat{A}_{i j}}{\operatorname{det} \widehat{M}}$, где $\hat{A}_{i j}-$ дополнительный минор к элементу $\widehat{\mu}_{j i}$ в матрице $\widehat{M}(\lambda)$, а $\widehat{\mu} \in \widehat{\mathcal{M}},\left.\widehat{\mu}\right|_{I}=1$ и $\operatorname{supp}(\widehat{\mu})$ достаточно мал. Из леммы 9.4 следует, что элементы матрицы $\widehat{M}^{-1}(\lambda)$ лежат в $\widehat{\mathcal{M}}$.

ЛЕмма 9.7. Пусть $\widehat{x}$ удовлетворяет системе уравнений ${ }^{9}$

$$
\widehat{M} \widehat{x}=0\left(\bmod \widehat{\mathcal{C}}_{0}^{n \star}\right),
$$

где $\widehat{M}-(n \times n)$-матрица с элементами $\widehat{\mu}_{i j} \in \widehat{\mathcal{M}}, \widehat{x} \in \widehat{\mathcal{C}}_{b}^{n}, \star \in\{+,-\}$. Пусть $\lambda_{0} \in \operatorname{sing} \operatorname{supp}^{\star}(\widehat{x})$. Тогдa $\operatorname{det} \widehat{M}\left(\lambda_{0}\right)=0$.

ДоказАТЕЛЬство. Выберем некоторый элемент $\widehat{\mu} \in \widehat{\mathcal{M}}$ с носителем в достаточно малом интервале $I, \lambda_{0} \in I, \widehat{\mu}\left(\lambda_{0}\right) \neq 0$. Домножим на него обе части системы (9.14). Получим

$$
\widehat{M}(\widehat{\mu} \widehat{x})=0\left(\bmod \widehat{\mathcal{C}}_{0}^{n \star}\right)
$$

Предположим, что $\operatorname{det} \widehat{M}\left(\lambda_{0}\right) \neq 0$. Согласно лемме 9.6 для матрицы $\widehat{M}(\lambda)$ на промежутке $I$ определена обратная матрица $\widehat{M}^{-1}(\lambda)$ с элементами из $\widehat{\mathcal{M}}$. Поэтому

$$
\widehat{\mu} \widehat{x}=0\left(\bmod \widehat{\mathcal{C}}_{0}^{n \star}\right),
$$

т. е. $\widehat{\mu} \widehat{x} \in \widehat{\mathcal{C}}_{0}^{n \star}$. Теперь возьмем достаточно малый интервал $J \subset I, \lambda_{0} \in J$, и произвольный элемент $\widehat{\mu}_{2} \in \widehat{\mathcal{M}}$, supp $\widehat{\mu}_{2} \subset J$. Согласно лемме 9.4 имеем $\widehat{\mu}_{2} \widehat{x}=\widehat{\mu}_{3} \widehat{\mu} \widehat{x}$ для некоторой $\widehat{\mu}_{3} \in \widehat{\mathcal{M}}$. Следовательно, $\widehat{\mu}_{2} \widehat{x} \in \widehat{\mathcal{C}}_{0}^{n \star}$. Это значит, что $\lambda_{0} \notin \operatorname{sing} \operatorname{supp}^{\star}(\widehat{x})$.

Лемма 9.8. Пусть $\widehat{x}$ удовлетворяет системе $(9.14)$, где $\operatorname{supp}\left(\widehat{x}_{l}\right) \in I, 1 \leqslant$ $l \leqslant n, I-$ достаточно мальй интервал. Пусть $\lambda_{0} \in I$, $\operatorname{det} \widehat{M}\left(\lambda_{0}\right)=0$, причем $\lambda_{0}-$ нуль порядка $k$. В остальных точках $\lambda$ интервала $I$ имеем $\operatorname{det} \widehat{M}(\lambda) \neq 0$. Тогда

$$
\widehat{x}=\sum_{i=1}^{k} \widehat{\sigma}_{i} \widehat{y}_{i}\left(\bmod \widehat{\mathcal{C}}_{0}^{n \star}\right),
$$

где $\widehat{\sigma}_{i} \in \widehat{\mathcal{M}}^{n}, \widehat{y}_{i} \in \widehat{\mathcal{C}}_{b}, a \widehat{\sigma}_{i}\left(\lambda_{0}\right) \in \operatorname{Ker} \widehat{M}\left(\lambda_{0}\right)$.

\footnotetext{
${ }^{9}$ Здесь и далее равенство по $\bmod \widehat{\mathcal{C}}_{0}^{n \star}$ означает равенство с точностью до функции из $\widehat{\mathcal{C}}_{0}^{n \star}$.
} 
ДокАзАтельство. Из равенства $\operatorname{Rank} \widehat{M}\left(\lambda_{0}\right)=n-k$ следует, что в матрице $\widehat{M}\left(\lambda_{0}\right)$ существует подматрица размера $(n-k) \times(n-k)$ с определителем, отличным от нуля. Не ограничивая общности, будем считать, что она находится в левом верхнем углу. Обозначим ее $\widehat{M}_{1}(\lambda)$. При достаточно малом $I$ в остальных точках $\lambda \in I$ ее определитель также отличен от нуля. Согласно лемме 9.6 на интервале $I$ к ней определена обратная матрица $\widehat{M}_{1}^{-1}$ с элементами из $\widehat{\mathcal{M}}$. Матрицу размера $(n-k) \times k$, стоящую в правом верхнем углу, обозначим $\widehat{M}_{2}(\lambda)$. Тогда выполняется следующее соотношение:

$$
\widehat{M}_{1} \widehat{x}^{n-k}=-\widehat{M}_{2} \widehat{x}^{k}\left(\bmod \widehat{\mathcal{C}}_{0}^{(n-k) \star}\right),
$$

где $\widehat{x}^{n-k}=\left(\widehat{x}_{1}, \ldots, x_{n-k}\right)^{T}, \quad \widehat{x}^{k}=\left(\widehat{x}_{n-k+1}, \ldots, \widehat{x}_{n}\right)^{T}$.

Перепишем равенство (9.15) в виде

$$
\widehat{x}^{n-k}=-\widehat{M}_{1}^{-1} \widehat{M}_{2} \widehat{x}^{k}\left(\bmod \widehat{\mathcal{C}}_{0}^{(n-k) \star}\right) \quad \text { или } \quad \widehat{x}=\sum_{i=1}^{k} \widehat{\sigma}_{i} \widehat{x}_{n-k+i}\left(\bmod \widehat{\mathcal{C}}_{0}^{n \star}\right),
$$

где $\widehat{\sigma}_{i}=\left(-\widehat{\psi}_{1 i}, \ldots,-\widehat{\psi}_{k i}, \mathbf{1}_{i}\right)^{T}, \widehat{\psi}_{i j}$ - элементы матрицы $\widehat{M}_{1}^{-1} \widehat{M}_{2}, \mathbf{1}_{i}$ - вектор длины $k$ со всеми компонентами, равными нулю, кроме единицы на $i$-м месте.

Таким образом, остается доказать следующее

УтверЖДЕниЕ 9.1. Выполнено $\widehat{\sigma}_{i}\left(\lambda_{0}\right) \in \operatorname{Ker} \widehat{M}\left(\lambda_{0}\right), 1 \leqslant i \leqslant n$.

ДокАЗАТЕЛЬство. В этом утверждении все функции подразумеваются взятыми в точке $\lambda_{0}$. Умножим матрицу $\widehat{M}$ слева на матрицу

$$
\left(\begin{array}{cc}
\widehat{M}_{1}^{-1} & 0 \\
0 & I_{k}
\end{array}\right)
$$

где $I_{k}-$ единичная матрица размера $k \times k$. Получим матрицу

$$
\left(\begin{array}{cc}
I_{n-k} & \widehat{M}_{1}^{-1} \widehat{M}_{2} \\
\cdots & \cdots
\end{array}\right)
$$

где многоточием обозначаются строки, линейно зависимые от первых $n-k$ строк. Матрица (9.16) невырождена, поэтому ядро матрицы (9.17) совпадет с ядром матрицы $\widehat{M}$. Прямым вычислением легко убедиться в том, что произведение матрицы (9.17) на вектор $\widehat{\sigma}_{i}\left(\lambda_{0}\right)$ равно нулю. Таким образом, утверждение и лемма доказаны.

\section{Список литературы}

1. D. Treschev, "Oscillator and thermostat", Discrete Contin. Dyn. Syst., 28:4 (2010), 1693-1712.

2. Н. Н. Боголюбов, "Элементарный пример установления статистического равновесия в системе, связанной с термостатом", О некоторых статистических методах в математической физике, АН УССР, Львов, 1945, 115-137. 
3. А.И. Комеч, "О стабилизации взаимодействия струны с нелинейным осциллятором", Вестн. Моск. ун-та. Сер. 1. Матем., мех., 1991, №6, 35-41; англ. пер.: A. I. Komech, "On the stabilization of interaction of a string with a nonlinear oscillator", Moscow Univ. Math. Bull., 46:6 (1992), 34-39.

4. A. Komech, H. Spohn, M. Kunze, "Long-time asymptotics for a classical particle interacting with a scalar wave field", Comm. Partial Differential Equations, 22:1-2 (1997), 307-335.

5. Al. Komech, An. Komech, "Global attractor for a nonlinear oscillator coupled to the Klein-Gordon field", Arch. Ration. Mech. Anal., 185:1 (2007), 105-142.

6. Al. Komech, An. Komech, "On global attraction to solitary waves for the Klein-Gordon field coupled to several nonlinear oscillators", J. Math. Pures Appl. (9), 93:1 (2010), 91-111.

7. Al. Komech, H. Spohn, "Long-time asymptotics for the coupled Maxwell-Lorentz equations", Comm. Partial Differential Equations, 25:3-4 (2000), 559-584.

8. Е. А. Горин, Д. В. Трещёв, "Относительный вариант теоремы Титчмарша о свертке", Функи. анализ и его прил., 46:1 (2012), 31-38; англ. пер.: Е. А. Gorin, D. V. Treschev, "Relative version of the Titchmarsh convolution theorem", Funct. Anal. Appl., 46:1 (2012), 26-32.

9. В.И. Богачёв, Основы теории меры, т. 1, РХД, Ижевск, 2006; англ. пер.: V. I. Bogachev, Measure theory, v. 1, Springer-Verlag, Berlin, 2007.

10. F. Riesz, B. Sz.-Nagy, Functional analysis, Dover Books Adv. Math., Dover Publ., New York, 1990.

11. И. М. Гельфанд, Лекиии по линейной алгебре, 5-е изд., МЦНМО, М., 1998; англ. пер. 4-го изд.: I. M. Gel'fand, Lectures on linear algebra, Dover Publ., New York, 1989.

12. В. С. Владимиров, Обобщенные функиии в математической физике, Наука, М., 1979; англ. пер.: V.S. Vladimirov, Generalized functions in mathematical physics, Mir, Moscow, 1979.

А. В. Дымов (А. V. Dymov)

Московский государственный университет

им. М. В. Ломоносова,

механико-математический факультет

E-mail: dendrr@yandex.ru
Поступило в редакцию 18.05 .2011

29.12.2011 